\title{
Synthesis and coordination chemistry of $\mathbf{N}$-doped polyphenylenes
}

\author{
S.D. Perera ${ }^{1}$, R.Quesada ${ }^{2}$ and S.M. Draper ${ }^{3}$ \\ ksper@ou.ac.lk
}

The $N$-doped polyphenylene derivatives are expected to show interesting optical, electrochemical and structural properties. 8-Azafluoranthene ligands (2a) and (2b) were prepared by a Diels-Alder cycloaddition reaction between 2-cyanopyridine and the corresponding

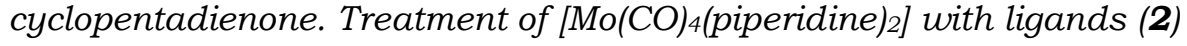
gave the tetracarbonylmolybdenum(O) complexes $\left[\mathrm{Mo}(\mathrm{CO})_{4}(\mathbf{2})\right](\mathbf{3})$, in which the ligand is coordinated to the metal through both nitrogen donors. Treatment of (2b) with [Pd(OAc) 2 ] gave the cyclometallated Pd(II) complex $[P d(O A c)(L)](\mathbf{4 a})$, in which $L$ is an anionic terdentate-ligand. Treatment of (2b) with $\left[\mathrm{PdCl}_{2}(\mathrm{NCPh})_{2}\right]$ gave the complex $[\mathrm{PdCl}(\mathrm{L})](\mathbf{4 b})$. The acetate group of (4a) can be easily replaced by halide ions to give [PdCl(L)] (4b), $[P d B r(L)]$ $(\mathbf{4 c})$ and $[P d I(L)](\mathbf{4 d})$, respectively. Treatment of $(\mathbf{4 a})$ with DMAP and $P P h_{3}$ in dichloromethane and the subsequent addition of $\mathrm{NH}_{4} P_{F_{6}}$ in methanol gave the corresponding salts $[P d(D M A P)(L)] P F_{6}(\mathbf{6} \boldsymbol{a})$ and $\left[P d\left(P P h_{3}\right)(L)\right] P F_{6}$ (6b), respectively.

\section{Introduction}

Polyphenylenes or hexaarylbenzene derivatives (I) are of considerable interest as they are the precursors for polycyclic aromatic hydrocarbons (Watson et al 2001). Introduction of heteroaromatic groups such as pyridyl and pyrimidyl into these systems opens the possibility for them to act as ligands. A few years ago we reported the synthesis of N-heterosuperbenzene (N-HSB) (II) by cyclodehydrogenation of the precursor (III) (Draper et al 2002). The Ndoped graphene (II) and its transition metal complexes have shown interesting optical, electrochemical and structural properties (Draper et al 2004). Recently we reported the synthesis of a pyridyl-centred polyphenylene (IV) and its coordination chemistry, particularly with rhodium and palladium (Ollagnier et al 2008). 
Figure 1 Some polyphenylene precursors and polycyclic aromatic hydrocarbons
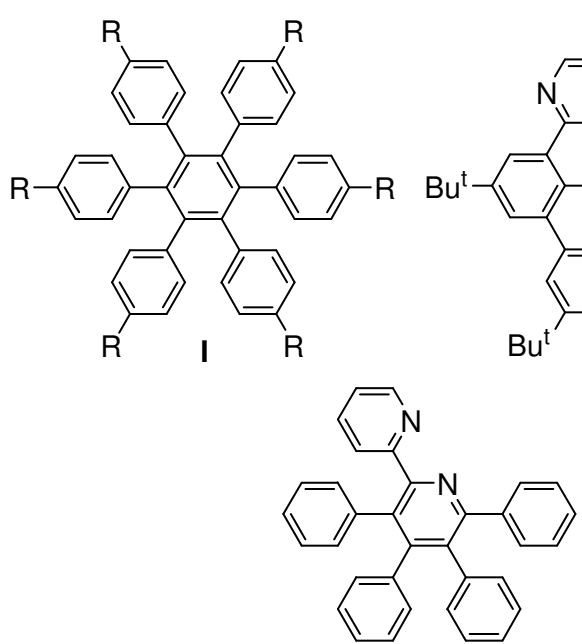

IV

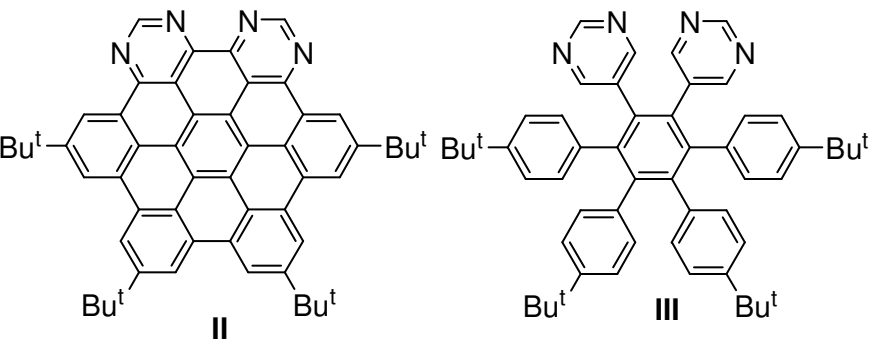

II<smiles></smiles>

v

Fluoranthene $(\mathbf{V})$ is a fused ring system with three benzene rings linked to a central 5-membered ring. The presence of a fused 5-membered ring in the skeleton makes fluoranthene derivatives suitable for the preparation of curved molecules such as corannulene and semibuckminsterfullerene (Sygula \& Rabidean, 1999). Fluoranthene derivatives can be cyclodehydrogenated to generate large aromatic sheets (Debad et al 1996 \& Wehmeier et al 2001). In this paper we describe the synthesis of new ligands (2a) and (2b) based on 8azafluoranthene, and their complexes with molybdenum(0) and palladium(II). (2a) and (2b) can be considered as bulky analogues of 6-phenyl-2,2'-bipyridine and they have the potential to coordinate to a metal centre in either bidentate or terdentate fashion via cyclometallation (see $\mathbf{A}$ and $\mathbf{B}$ in Figure 2). 
Figure 2 Coordination modes of (2) to a metal centre $\mathrm{M} . \mathrm{R}=\mathrm{H}$ or $\mathrm{Bu}^{\mathrm{t}}$.

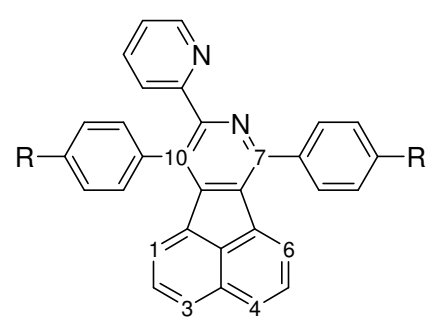

(2)

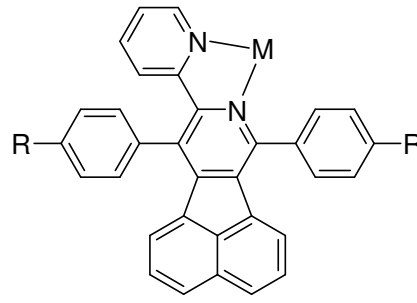

A $\mathrm{M}(\mathrm{LH})$

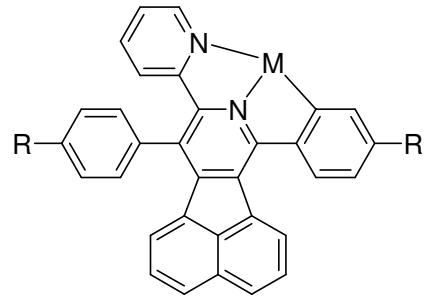

B $\mathrm{M}(\mathrm{L})$

\section{Methodology}

All the experiments were carried out in an inert atmosphere (nitrogen or argon). Elemental analyses were carried out on a Carlo Erba 1006 automatic analyser. IR spectra were recorded on a PerkinElmer Spectrum One FT-IR spectrometer fitted with a universal ATR sampling accessory. Mass spectral data were obtained using a micromass LCT electrospray mass spectrometer. NMR spectra were recorded on a Bruker DPX 400 spectrometer (operating frequencies for ${ }^{1} \mathrm{H}$ and ${ }^{13} \mathrm{C}$ are 400.13 and $100.62 \mathrm{MHz}$, respectively) or Bruker Avance $\Pi 600$ spectrometer (operating frequencies for ${ }^{1} \mathrm{H}$ and ${ }^{13} \mathrm{C}$ are 600.13 and $150.9 \mathrm{MHz}) .{ }^{1} \mathrm{H}$ and ${ }^{13} \mathrm{C}$ chemical shifts $(\delta)$ are in ppm with respect to TMS and coupling constants $(\mathrm{J})$ are in $\mathrm{Hz}$. Flash chromatography was carried out using silica gel as the stationary phase.

Acenaphthenequinone was purchased from Aldrich. 7,9-Diphenyl-8Hcyclopenta[1] acenaphthylen-8-one (1a) was prepared according to a literature procedure (Wehmeier et al 2001).

\section{7,9-Bis(4-tert-butylphenyl)-8H-cyclopenta[l]acenaphthylen-8-one (1b)}

Acenaphthenequinone $(400 \mathrm{mg}, 2.19 \mathrm{mmol}), \quad$ 1,3-bis(4-tertbutylphenyl)propan-2-one (700 $\mathrm{mg}, 2.2 \mathrm{mmol})$ and $\mathrm{NaOH}(100 \mathrm{mg})$ were stirred at room temperature in $\mathrm{MeOH}(50 \mathrm{~mL})$ for $24 \mathrm{~h}$. The resulting black precipitate (1b) was filtered, washed with $\mathrm{MeOH}$ and dried in a vacuum. Yield (960 $\mathrm{mg}, 94 \%$ ). An analytical sample was crystallised from $\mathrm{CH}_{2} \mathrm{Cl}_{2} / \mathrm{MeOH}$. Found: C, 88.69; H, 6.79, calcd. (\%) for $\mathrm{C}_{35} \mathrm{H}_{32} \mathrm{O} \cdot 0.1 \mathrm{CH}_{2} \mathrm{Cl}_{2}$ : C, 88.28; $\mathrm{H}, 6.80$. IR (neat) $\mathrm{cm}^{-1}: 2958,1701$, 1473, 1360, 1271, 1130, 840, 825 and 773. ${ }^{1} \mathrm{H}-\mathrm{NMR}(400 \mathrm{MHz}$, 
$\left.\mathrm{CDCl}_{3}\right): 8.12\left(\mathrm{~d}, 2 \mathrm{H},{ }^{3} \mathrm{~J}(\mathrm{HH}) 7.0 \mathrm{~Hz}, \mathrm{H}^{3}\right), 7.88\left(\mathrm{~d}, 2 \mathrm{H},{ }^{3} \mathrm{~J}(\mathrm{HH}) 8.0, \mathrm{~Hz}, \mathrm{H}^{1}\right)$, $7.80\left(\mathrm{~d}, 4 \mathrm{H},{ }^{3} \mathrm{~J}(\mathrm{HH}) 8.0 \mathrm{~Hz}, \mathrm{H}^{4}\right), 7.61$ (app. t, $2 \mathrm{H}, 3 \mathrm{~J}(\mathrm{HH}) 7.5 \mathrm{~Hz}, \mathrm{H}^{2}$ ), $7.57\left(\mathrm{~d}, 4 \mathrm{H},{ }^{3} \mathrm{~J}(\mathrm{HH}) 8.0 \mathrm{~Hz}, \mathrm{H}^{5}\right)$ and $1.42\left(\mathrm{~s}, 18 \mathrm{H}, \mathrm{CMe}_{3}\right) .{ }^{13} \mathrm{C}-\mathrm{NMR}(100$ $\left.\mathrm{MHz}, \mathrm{CDCl}_{3}\right): 202.0(1 \mathrm{C}, \mathrm{C}=\mathrm{O}), 128.3\left(2 \mathrm{C}, \mathrm{C}^{4}\right), 127.9\left(1 \mathrm{C}, \mathrm{C}^{2}\right), 127.0$ (1C, $\left.\mathrm{C}^{1}\right), 125.1$ (2C, $\left.\mathrm{C}^{5}\right), 121.1$ ( $\left.\mathrm{C}_{\text {quat }}\right), 120.4\left(1 \mathrm{C}, \mathrm{C}^{3}\right), 34.4\left(\mathrm{CMe}_{3}\right)$ and $30.9\left(\mathrm{CMe}_{3}\right)$.

\section{7,10-Diphenyl-9-(2-pyridyl)-8-azafluoranthene (2a)}

7,9-Diphenyl-8H-cyclopenta[1]acenaphthylen-8-one $(600 \mathrm{mg}$, $1.68 \mathrm{mmol})$ and 2-cyano pyridine $(1.1 \mathrm{~g}, 11.6 \mathrm{mmol})$ were refluxed under nitrogen for $48 \mathrm{~h}$. The resulting brownish red solution was allowed to cool. Chromatography on silica using $\mathrm{MeOH} / \mathrm{CH}_{2} \mathrm{Cl}_{2}$ gave (2a) as a pale yellow crystalline solid. Yield (180 mg, 25\%). Found: C, 87.30; $\mathrm{H}, 4.62 ; \mathrm{N}, 6.23$, calcd. (\%) for $\mathrm{C}_{32} \mathrm{H}_{20} \mathrm{~N}_{2} .0 \cdot 1 \mathrm{CH}_{2} \mathrm{Cl}_{2}: \mathrm{C}, 87.35 ; \mathrm{H}$, 4.58; N, 6.35. IR (neat) $\mathrm{cm}^{-1}$ : 3006, 1586, 1546, 1475, 1422, 1402, 1276, 1261, 828, 766, 749 and 704. ESI-MS (acetone, $\mathrm{m} / \mathrm{z}$ ): found: 433.1697, calcd. 433.1705, for $\mathrm{C}_{32} \mathrm{H}_{21} \mathrm{~N}_{2}$, [M+1] ${ }^{+}$. ${ }^{1} \mathrm{H}-\mathrm{NMR}(400 \mathrm{MHz}$, $\left.\mathrm{CDCl}_{3}\right): 8.52\left(\mathrm{br}, \mathrm{m}, 1 \mathrm{H},{ }^{3} \mathrm{~J}(\mathrm{HH}) 5.0 \mathrm{~Hz}, \mathrm{H}^{10}\right), 7.95\left(\mathrm{~m}, 2 \mathrm{H}, \mathrm{H}^{4}\right), 7.91(\mathrm{~d}$, $\left.1 \mathrm{H},{ }^{3} \mathrm{~J}(\mathrm{HH}) 8.0 \mathrm{~Hz}, \mathrm{H}^{1}\right), 7.87\left(\mathrm{~d}, 1 \mathrm{H},{ }^{3} \mathrm{~J}(\mathrm{HH}) 8.0 \mathrm{~Hz}, \mathrm{H}^{1}\right), 7.63-7.55(\mathrm{~m}$, $5 \mathrm{H}, \mathrm{H}^{3}, \mathrm{H}^{5}, \mathrm{H}^{6}$ and $\left.\mathrm{H}^{8}\right), 7.52-7.49\left(\mathrm{~m}, 2 \mathrm{H}, \mathrm{H}^{2}\right.$ and $\left.\mathrm{H}^{7}\right), 7.46-7.40(\mathrm{~m}$, $6 \mathrm{H}, \mathrm{H}^{2}, \mathrm{H}^{4}, \mathrm{H}^{5}$ and $\left.\mathrm{H}^{6}\right), 7.12\left(\mathrm{~m}, 1 \mathrm{H},{ }^{3} \mathrm{~J}(\mathrm{HH}) 5.0,7.5 \mathrm{~Hz},{ }^{4} \mathrm{~J}(\mathrm{HH}) 1.5 \mathrm{~Hz}\right.$, $\left.\mathrm{H}^{9}\right)$ and $7.00\left(\mathrm{~d}, 1 \mathrm{H},{ }^{3} \mathrm{~J}(\mathrm{HH}) 7.0, \mathrm{~Hz}, \mathrm{H}^{3}\right) .{ }^{13} \mathrm{C}-\mathrm{NMR}\left(100 \mathrm{MHz}, \mathrm{CDCl}_{3}\right)$ : $148.4\left(1 \mathrm{C}, \mathrm{C}^{10}\right), 135.2\left(1 \mathrm{C}, \mathrm{C}^{8}\right), 129.6\left(2 \mathrm{C}, \mathrm{C}^{4}\right), 128.8\left(2 \mathrm{C}, \mathrm{C}^{4}\right), 128.5$ $\left(1 \mathrm{C}, \mathrm{C}^{1}\right), 128.3\left(1 \mathrm{C}, \mathrm{C}^{6}\right), 128.2\left(2 \mathrm{C}, \mathrm{C}^{5}\right), 128.1$ (2C, $\left.\mathrm{C}^{5}\right), 127.5\left(1 \mathrm{C}, \mathrm{C}^{6}\right)$, $127.3\left(1 \mathrm{C}, \mathrm{C}^{2}\right), 127.2\left(1 \mathrm{C}, \mathrm{C}^{2}\right), 127.0\left(1 \mathrm{C}, \mathrm{C}^{1}\right), 124.9\left(1 \mathrm{C}, \mathrm{C}^{3}\right), 124.6$ $\left(1 \mathrm{C}, \mathrm{C}^{7}\right), 123.4\left(1 \mathrm{C}, \mathrm{C}^{3}\right)$ and $121.6\left(1 \mathrm{C}, \mathrm{C}^{9}\right)$.

\section{7,10-Di(4-tert-butylphenyl)-9-(2-pyridyl)-8-azafluoranthene (2b) (LH)}

Bis(4-tert-butylphenyl)-8H-cyclopenta[1]acenaphthylen-8-one (700 mg, $1.49 \mathrm{mmol})$ and 2-cyanopyridine $(2.0 \mathrm{~g})$ were refluxed under nitrogen for $60 \mathrm{~h}$. The resulting brownish red solution was allowed to cool. Chromatography on silica using $\mathrm{MeOH}$ and $\mathrm{CH}_{2} \mathrm{Cl}_{2}$ gave $(\mathbf{2 b})$ as a pale yellow crystalline solid. Yield (310 mg, 38\%). Found: C, 88.69; H, 6.79, calcd. (\%) for $\mathrm{C}_{35} \mathrm{H}_{32} \mathrm{O} \cdot 0.1 \mathrm{CH}_{2} \mathrm{Cl}_{2}$ : C, 88.28; $\mathrm{H}, 6.80$. IR (neat) $\mathrm{cm}^{-1}$ : 2958, 1701, 1473, 1360, 1271, 1130, 840, 825 and 773. ESI-MS (acetone, $\mathrm{m} / z$ ): found: 545.2953, calcd. 545.2957, for $\mathrm{C}_{40} \mathrm{H}_{37} \mathrm{~N}_{2},[\mathrm{M}+1]^{+}$. ${ }^{1} \mathrm{H}-\mathrm{NMR}\left(400 \mathrm{MHz}, \mathrm{CDCl}_{3}\right): 8.52\left(\mathrm{br}, \mathrm{m}, 1 \mathrm{H},{ }^{3} \mathrm{~J}(\mathrm{HH}) 4.0 \mathrm{~Hz}, \mathrm{H}^{10}\right), 7.91$ (d, $\left.1 \mathrm{H}, 3 \mathrm{~J}(\mathrm{HH}) 8.0 \mathrm{~Hz}, \mathrm{H}^{1}\right), 7.90\left(\mathrm{~d}, 2 \mathrm{H}, 3 \mathrm{~J}(\mathrm{HH}) 8.5 \mathrm{~Hz}, \mathrm{H}^{4}\right), 7.87(\mathrm{~d}, 1 \mathrm{H}$, 3J(HH) $\left.8.0 \mathrm{~Hz}, \mathrm{H}^{1}\right), 7.72\left(\mathrm{~d}, 1 \mathrm{H}, 3 \mathrm{~J}(\mathrm{HH}) 7.0 \mathrm{~Hz}, \mathrm{H}^{3}\right), 7.61$ (d, 2H, 3J(HH) $\left.8.5 \mathrm{~Hz}, \mathrm{H}^{5}\right), 7.55-7.51\left(\mathrm{~m}, 2 \mathrm{H}, \mathrm{H}^{2}\right.$ and $\left.\mathrm{H}^{8}\right), 7.47-7.44\left(\mathrm{~m}, 3 \mathrm{H},{ }^{3} \mathrm{~J}(\mathrm{HH})\right.$ 
$8.5 \mathrm{~Hz}, \mathrm{H}^{5}$ and $\left.\mathrm{H}^{2}\right), 7.43\left(\mathrm{~d}, 1 \mathrm{H},{ }^{3} \mathrm{~J}(\mathrm{HH}) 8.0 \mathrm{~Hz}, \mathrm{H}^{7}\right), 7.35\left(\mathrm{~d}, 2 \mathrm{H},{ }^{3} \mathrm{~J}(\mathrm{HH})\right.$ $\left.8.5 \mathrm{~Hz}, \mathrm{H}^{4}\right), 7.12\left(\mathrm{~m}, 1 \mathrm{H}, \mathrm{H}^{9}\right), 7.02\left(\mathrm{~d}, 1 \mathrm{H}, 3 \mathrm{~J}(\mathrm{HH}) 7.0, \mathrm{~Hz}, \mathrm{H}^{3}\right), 1.45(\mathrm{~s}$, 9H, $\left.\mathrm{CMe}_{3}\right)$ and $1.41\left(\mathrm{~s}, 9 \mathrm{H}, \mathrm{CMe}_{3}\right) .{ }^{13} \mathrm{C}-\mathrm{NMR}\left(100 \mathrm{MHz}, \mathrm{CDCl}_{3}, \delta\right.$ in ppm): $148.3\left(1 \mathrm{C}, \mathrm{C}^{10}\right), 134.9\left(1 \mathrm{C}, \mathrm{C}^{8}\right), 129.2\left(2 \mathrm{C}, \mathrm{C}^{4}\right), 128.4\left(2 \mathrm{C}, \mathrm{C}^{4}\right)$, $128.3\left(1 \mathrm{C}, \mathrm{C}^{1}\right), 127.5\left(1 \mathrm{C}, \mathrm{C}^{2}\right), 127.3\left(1 \mathrm{C}, \mathrm{C}^{2}\right), 126.9\left(1 \mathrm{C}, \mathrm{C}^{1}\right), 125.0$ (2C, $\left.\mathrm{C}^{5}\right), 124.9$ (2C, C5), 124.8 (1C, $\left.\mathrm{C}^{3}\right), 124.6$ (1C, $\left.\mathrm{C}^{7}\right), 123.4\left(1 \mathrm{C}, \mathrm{C}^{3}\right)$, $121.5\left(1 \mathrm{C}, \mathrm{C}^{9}\right), 34.4\left(1 \mathrm{C}, \mathrm{CMe}_{3}\right), 34.2\left(1 \mathrm{C}, \mathrm{CMe}_{3}\right)$ and $31.0\left(6 \mathrm{C}, \mathrm{CMe}_{3}\right)$.

\section{$\left[\mathrm{Mo}(\mathrm{CO})_{4}(2 a)\right](3 a)$}

A solution containing (2a) $(20 \mathrm{mg}, 0.046 \mathrm{mmol})$ and $\left[\mathrm{Mo}(\mathrm{CO})_{4} \text { (piperidine) }\right)_{2}$ (17 $\left.\mathrm{mg}, 0.046 \mathrm{mmol}\right)$ in $\mathrm{CH}_{2} \mathrm{Cl}_{2}(1.5 \mathrm{~mL})$ was stirred at room temperature for $30 \mathrm{~min}$. The resulting dark brown solution was concentrated to a low volume $(\mathrm{ca} .0 .5 \mathrm{~mL})$; the addition of methanol gave (3a) as black crystals (29 mg, 97\%). Found: C, 57.99; H, 3.02; N, 3.62, calcd. (\%) for $\mathrm{C}_{36} \mathrm{H}_{20} \mathrm{~N}_{2} \mathrm{O}_{4} \mathrm{Mo} \cdot 1.5 \mathrm{CH}_{2} \mathrm{Cl}_{2}$ : C, 58.70; $\mathrm{H}$, 3.01; N, 3.64. IR (neat, $v, \mathrm{~cm}^{-1}$ ): 2005, 1863, 1823, 1420, 1408, 828, 766 and 700. ${ }^{1} \mathrm{H}-\mathrm{NMR}\left(400 \mathrm{MHz}, \mathrm{CDCl}_{3}\right): 9.24\left(\mathrm{br}, \mathrm{d}, 1 \mathrm{H},{ }^{3} \mathrm{~J}(\mathrm{HH}) 5.5\right.$ $\left.\mathrm{Hz}, \mathrm{H}^{10}\right), 7.98\left(\mathrm{~d}, 1 \mathrm{H},{ }^{3} \mathrm{~J}(\mathrm{HH}) 8.0 \mathrm{~Hz}, \mathrm{H}^{1}\right.$ or $\left.\mathrm{H}^{1}\right), 7.91\left(\mathrm{~d}, 1 \mathrm{H},{ }^{3} \mathrm{~J}(\mathrm{HH}) 8.5\right.$ $\mathrm{Hz}, \mathrm{H}^{1}$ or $\left.\mathrm{H}^{1}\right), 7.75-7.71(\mathrm{~m}, 6 \mathrm{H}, \mathrm{Ph}), 7.67-7.65(\mathrm{~m}, 2 \mathrm{H}, \mathrm{Ph}), 7.59-7.57$ (m, 2H, Ph), 7.48-7.44 (m, 2H, $\mathrm{H}^{2}$ and $\left.\mathrm{H}^{2}\right), 7.41\left(\mathrm{~m}, 1 \mathrm{H},{ }^{3} \mathrm{~J}(\mathrm{HH}) 7.5 \mathrm{~Hz}\right.$, $\left.{ }^{4} \mathrm{~J}(\mathrm{HH}) 1.5 \mathrm{~Hz}, \mathrm{H}^{8}\right), 7.23\left(\mathrm{~d}, 1 \mathrm{H},{ }^{3} \mathrm{~J}(\mathrm{HH}) 8.5 \mathrm{~Hz}, \mathrm{H}^{7}\right), 7.16\left(\mathrm{~m}, 1 \mathrm{H},{ }^{3} \mathrm{~J}(\mathrm{HH})\right.$ $\left.5.5 \mathrm{~Hz},{ }^{4} \mathrm{~J}(\mathrm{HH}) 1.5 \mathrm{~Hz}, \mathrm{H}^{9}\right), 6.79\left(\mathrm{~d}, 1 \mathrm{H},{ }^{3} \mathrm{~J}(\mathrm{HH}) 7.5 \mathrm{~Hz}, \mathrm{H}^{3}\right.$ or $\left.\mathrm{H}^{3}\right)$ and $6.66\left(\mathrm{~d}, 1 \mathrm{H},{ }^{3} \mathrm{~J}(\mathrm{HH}) 7.0 \mathrm{~Hz}, \mathrm{H}^{3}\right.$ or $\left.\mathrm{H}^{3}\right) .{ }^{13} \mathrm{C}-\mathrm{NMR}\left(100 \mathrm{MHz}, \mathrm{CDCl}_{3}\right)$ : $225.7(1 \mathrm{C}, \mathrm{C} \equiv \mathrm{O}), 217.1(1 \mathrm{C}, \mathrm{C} \equiv \mathrm{O}), 204.6(2 \mathrm{C}, \mathrm{C} \equiv \mathrm{O}), 152.5\left(1 \mathrm{C}, \mathrm{C}{ }^{10}\right)$, 134.9 (1C, $\left.\mathrm{C}^{8}\right), 130.0$ (1C, Ph), 129.7 (1C, $\mathrm{C}^{1}$ or $\mathrm{C}^{1}$ ), 129.4 (2C, Ph), 129.1 (1C, Ph), 129.0 (2C, Ph), 128.9 (2C, Ph), 128.4 (2C, Ph), 128.2 (1C, $\mathrm{C}^{1}$ or $\mathrm{C}^{1}$ ), 127.9 (1C, $\mathrm{C}^{2}$ or $\left.\mathrm{C}^{2}\right), 127.6\left(1 \mathrm{C}, \mathrm{C}^{2}\right.$ or $\left.\mathrm{C}^{2}\right), 126.9\left(1 \mathrm{C}, \mathrm{C}^{7}\right)$, $126.3\left(1 \mathrm{C}, \mathrm{C}^{3}\right.$ or $\left.\mathrm{C}^{3}\right), 125.4\left(1 \mathrm{C}, \mathrm{C}^{3}\right.$ or $\left.\mathrm{C}^{3}\right)$ and $122.9\left(1 \mathrm{C}, \mathrm{C}^{9}\right)$.

\section{$\left[\mathrm{Mo}(\mathrm{CO})_{4}(2 \mathrm{~b})\right](3 b)$}

A solution containing (2b) $(20 \mathrm{mg}, 0.036 \mathrm{mmol})$ and $\left.\left[\mathrm{Mo}(\mathrm{CO})_{4} \text { (piperidine) }\right)_{2}\right](13.8 \mathrm{mg}, 0.036 \mathrm{mmol})$ in $\mathrm{CH}_{2} \mathrm{Cl}_{2}(2 \mathrm{~mL})$ was stirred at $20 \circ \mathrm{C}$ for $30 \mathrm{~min}$. The resulting dark brown solution was concentrated to a low volume $(c a .0 .5 \mathrm{~mL})$, then $\mathrm{MeOH}$ was added to give (3b) as black crystals (23 mg, 83\%). Found: C, 69.87; H, 4.81; N, 3.65, calcd. (\%) for $\mathrm{C}_{44} \mathrm{H}_{36} \mathrm{~N}_{2} \mathrm{O}_{4} \mathrm{Mo}$ : C, 70.21; H, 4.82; N, 3.72. IR (neat, $\left.v, \mathrm{~cm}^{-1}\right): 2963,2001,1872,1829,1815,1422,1118,828$ and $779 .{ }^{1} \mathrm{H}-$ NMR (400 MHz, $\left.\mathrm{CDCl}_{3}\right): 9.23\left(\mathrm{br}, \mathrm{d}, 1 \mathrm{H},{ }^{3} \mathrm{~J}(\mathrm{HH}) 4.5 \mathrm{~Hz}, \mathrm{H}^{10}\right), 7.97$ (d, $1 \mathrm{H},{ }^{3} \mathrm{~J}(\mathrm{HH}) 8.0 \mathrm{~Hz}, \mathrm{H}^{1}$ or $\left.\mathrm{H}^{1}\right), 7.90\left(\mathrm{~d}, 1 \mathrm{H},{ }^{3} \mathrm{~J}(\mathrm{HH}) 8.0 \mathrm{~Hz}, \mathrm{H}^{1}\right.$ or $\left.\mathrm{H}^{1}\right)$, $7.73\left(\mathrm{~d}, 2 \mathrm{H},{ }^{3} \mathrm{~J}(\mathrm{HH}) 8.5 \mathrm{~Hz}, \mathrm{H}^{5}\right.$ or $\left.\mathrm{H}^{5}\right), 7.71\left(\mathrm{~d}, 2 \mathrm{H},{ }^{3} \mathrm{~J}(\mathrm{HH}) 8.5 \mathrm{~Hz}, \mathrm{H}^{5}\right.$ or $\left.\mathrm{H}^{5}\right), 7.58\left(\mathrm{~d}, 2 \mathrm{H},{ }^{3} \mathrm{~J}(\mathrm{HH}) 8.5 \mathrm{~Hz}, \mathrm{H}^{4}\right.$ or $\left.\mathrm{H}^{4}\right), 7.47\left(\mathrm{~d}, 2 \mathrm{H},{ }^{3} \mathrm{~J}(\mathrm{HH}) 8.5 \mathrm{~Hz}\right.$, $\mathrm{H}^{4}$ or $\left.\mathrm{H}^{4}\right), 7.48-7.44\left(\mathrm{~m}, 2 \mathrm{H}, \mathrm{H}^{2}\right.$ and $\left.\mathrm{H}^{2}\right), 7.36\left(\mathrm{~m}, 1 \mathrm{H},{ }^{3} \mathrm{~J}(\mathrm{HH}) 8.5 \mathrm{~Hz}\right.$, 
$\left.{ }^{4} \mathrm{~J}(\mathrm{HH}) 1.5 \mathrm{~Hz}, \mathrm{H}^{8}\right), 7.20\left(\mathrm{~d}, 1 \mathrm{H},{ }^{3} \mathrm{~J}(\mathrm{HH}) 8.0 \mathrm{~Hz}, \mathrm{H}^{7}\right), 7.13\left(\mathrm{br}, \mathrm{m}, 1 \mathrm{H}, \mathrm{H}^{9}\right)$, $6.81\left(\mathrm{~d}, 1 \mathrm{H},{ }^{3} \mathrm{~J}(\mathrm{HH}) 7.5 \mathrm{~Hz}, \mathrm{H}^{3}\right.$ or $\left.\mathrm{H}^{3}\right), 6.72\left(\mathrm{~d}, 1 \mathrm{H},{ }^{3} \mathrm{~J}(\mathrm{HH}) 7.0 \mathrm{~Hz}, \mathrm{H}^{3}\right.$ or $\left.\mathrm{H}^{3}\right), 1.52\left(\mathrm{~s}, 9 \mathrm{H}, \mathrm{CMe}_{3}\right)$ and $1.51\left(\mathrm{~s}, 9 \mathrm{H}, \mathrm{CMe}_{3}\right) .{ }^{13} \mathrm{C}-\mathrm{NMR}: 225.8(1 \mathrm{C}$, $\mathrm{C} \equiv \mathrm{O}), 216.6(1 \mathrm{C}, \mathrm{C} \equiv \mathrm{O}), 204.8(2 \mathrm{C}, \mathrm{C} \equiv \mathrm{O}), 152.4$ (1C, $\left.\mathrm{C}^{10}\right), 134.7$ (1C, $\left.\mathrm{C}^{8}\right), 129.7\left(1 \mathrm{C}, \mathrm{C}^{1}\right.$ or $\mathrm{C}^{1}$ ), $128.6(\mathrm{Ar}), 128.0\left(1 \mathrm{C}, \mathrm{C}^{1}\right.$ or $\left.\mathrm{C}^{1}\right), 127.9(\mathrm{Ar}$ and $\mathrm{C}^{2}$ or $\mathrm{C}^{2}$ ), $127.5\left(1 \mathrm{C}, \mathrm{C}^{2}\right.$ or $\left.\mathrm{C}^{2}\right), 126.8\left(1 \mathrm{C}, \mathrm{C}^{7}\right), 126.8(\mathrm{Ar}), 126.3$ (1C, $\mathrm{C}^{3}$ or $\left.\mathrm{C}^{3}\right)$, $125.7(\mathrm{Ar}), 125.4\left(1 \mathrm{C}, \mathrm{C}^{3}\right.$ or $\left.\mathrm{C}^{3}\right), 122.7\left(1 \mathrm{C}, \mathrm{C}^{9}\right), 34.61$ (1C, $\left.\mathrm{CMe}_{3}\right), 34.58$ (1C, $\left.\mathrm{CMe}_{3}\right), 31.1(3 \mathrm{C}, \mathrm{CMe})$ and $31.0(3 \mathrm{C}, \mathrm{CMe})$.

\section{$[P d(O A c)(L)](4 a)$}

A solution containing $(\mathbf{2 b})(40 \mathrm{mg}, 0.073 \mathrm{mmol})$ and $\left[\mathrm{Pd}(\mathrm{OAc})_{2}\right](16.5$ $\mathrm{mg}, 0.073 \mathrm{mmol})$ in $\mathrm{CH}_{2} \mathrm{Cl}_{2}(4 \mathrm{~mL})$ was refluxed for $3 \mathrm{~h}$. The solution was concentrated to a low volume $(c a .1 \mathrm{~mL})$ then diethyl ether was added to give (4a) as a yellow solid (49 mg, 94\%). Found: C, 69.33; H, 5.13; N 3.69, calcd. (\%) for $\mathrm{C}_{42} \mathrm{H}_{38} \mathrm{~N}_{2} \mathrm{O}_{2} \mathrm{Pd} \cdot 0.2 \mathrm{CH}_{2} \mathrm{Cl}_{2}$ : C, 69.79; H, 5.33; N 3.86. IR (neat, $v, \mathrm{~cm}^{-1}$ ): 2962, $1619(\mathrm{C}=\mathrm{O}), 1584,1420,1368,1319$, 1266, 824, 781 and 669. ESI-MS (acetone, $\mathrm{m} / \mathrm{z}$ ): found: 649.1848, calcd. 649.1835 for $\mathrm{C}_{40} \mathrm{H}_{35} \mathrm{~N}_{2} \mathrm{Pd}$, [M-OAc] ${ }^{+} .{ }^{1} \mathrm{H}-\mathrm{NMR}\left(400 \mathrm{MHz}, \mathrm{CDCl}_{3}, \delta\right.$ in $\mathrm{ppm}): 8.81\left(\mathrm{~d}, 1 \mathrm{H},{ }^{3} \mathrm{~J}(\mathrm{HH}) 7.0 \mathrm{~Hz}, \mathrm{H}^{3}\right), 8.61\left(\mathrm{br}, \mathrm{d}, 1 \mathrm{H},{ }^{3} \mathrm{~J}(\mathrm{HH}) 5.0 \mathrm{~Hz}\right.$, $\left.\mathrm{H}^{10}\right), 8.21\left(\mathrm{~d}, 1 \mathrm{H},{ }^{3} \mathrm{~J}(\mathrm{HH}) 8.0 \mathrm{~Hz}, \mathrm{H}^{13}\right), 8.02\left(\mathrm{~d}, 1 \mathrm{H},{ }^{3} \mathrm{~J}(\mathrm{HH}) 7.5 \mathrm{~Hz}, \mathrm{H}^{1}\right)$, $8.02\left(\mathrm{~d}, 1 \mathrm{H},{ }^{3} \mathrm{~J}(\mathrm{HH}) 7.5 \mathrm{~Hz}, \mathrm{H}^{1}\right), 7.77\left(\mathrm{t}, 1 \mathrm{H},{ }^{3} \mathrm{~J}(\mathrm{HH}) 7.5 \mathrm{~Hz}, \mathrm{H}^{2}\right), 7.74(\mathrm{~d}$, 2H, $\left.{ }^{3} \mathrm{~J}(\mathrm{HH}) 8.0 \mathrm{~Hz}, \mathrm{H}^{5}\right), 7.47\left(\mathrm{~d}, 1 \mathrm{H},{ }^{4} \mathrm{~J}(\mathrm{HH}) 1.5 \mathrm{~Hz}, \mathrm{H}^{11}\right), 7.47$ (m, 1H, ${ }^{4} \mathrm{~J}(\mathrm{HH}) 1.5 \mathrm{~Hz}, \mathrm{H}^{8}$, overlaps with $\left.\mathrm{H}^{11}\right), 7.40\left(\mathrm{~m}, 1 \mathrm{H}, \mathrm{H}^{2}\right.$, overlaps with $\left.\mathrm{H}^{4}\right), 7.40\left(\mathrm{~d}, 2 \mathrm{H},{ }^{3} \mathrm{~J}(\mathrm{HH}) 8.0 \mathrm{~Hz}, \mathrm{H}^{4}\right), 7.35\left(\mathrm{~m}, 1 \mathrm{H}, \mathrm{H}^{9}\right), 7.26(\mathrm{dd}, 1 \mathrm{H}$, $\left.{ }^{3} \mathrm{~J}(\mathrm{HH}) 8.0 \mathrm{~Hz},{ }^{4} \mathrm{~J}(\mathrm{HH}) 1.5 \mathrm{~Hz}, \mathrm{H}^{12}\right), 6.83\left(\mathrm{~d}, 1 \mathrm{H},{ }^{3} \mathrm{~J}(\mathrm{HH}) 8.5 \mathrm{~Hz}, \mathrm{H}^{7}\right), 6.46$ (d, $\left.1 \mathrm{H}, 3 \mathrm{~J}(\mathrm{HH}) 7.0 \mathrm{~Hz}, \mathrm{H}^{3}\right), 1.53\left(\mathrm{~s}, 9 \mathrm{H}, \mathrm{CMe}_{3}\right)$ and $1.43\left(\mathrm{~s}, 9 \mathrm{H}, \mathrm{CMe}_{3}\right)$. ${ }^{13} \mathrm{C}-\mathrm{NMR}: 177.5(\mathrm{C}=\mathrm{O}), 160.4\left(\mathrm{C}_{\text {quat }}, \mathrm{PdC}\right), 149.8\left(1 \mathrm{C}, \mathrm{C}^{10}\right), 137.6(1 \mathrm{C}$, $\left.\mathrm{C}^{8}\right), 129.9$ (1C, $\left.\mathrm{C}^{11}\right), 129.6\left(1 \mathrm{C}, \mathrm{C}^{1}\right), 128.4\left(1 \mathrm{C}, \mathrm{C}^{1}\right), 128.2\left(2 \mathrm{C}, \mathrm{C}^{4}\right)$, 127.6 (1C, $\left.\mathrm{C}^{2}\right), 127.5$ (1C, $\left.\mathrm{C}^{2}\right), 127.1$ (2C, C5), 126.2 (1C, C13), 125.6 $\left(1 \mathrm{C}, \mathrm{C}^{3}\right), 125.6\left(1 \mathrm{C}, \mathrm{C}^{3}\right), 125.3$ (1C, C7), 125.1 (1C, C9), 120.9 (1C, C12), $34.71\left(\mathrm{CMe}_{3}\right), 34.65\left(\mathrm{CMe}_{3}\right), 31.0\left(\mathrm{CMe}_{3}\right), 30.8\left(\mathrm{CMe}_{3}\right)$ and 23.8 $(\mathrm{C}(=\mathrm{O}) M e)$.

\section{$[\operatorname{PdCl}(L)](4 b)$}

A solution containing the ligand $(\mathbf{2 b})(20 \mathrm{mg}, 0.037 \mathrm{mmol})$ and $\left[\mathrm{PdCl}_{2}(\mathrm{NCPh})_{2}\right](14 \mathrm{mg}, 0.037 \mathrm{mmol})$ in $\mathrm{CH}_{2} \mathrm{Cl}_{2}(2 \mathrm{~mL})$ was refluxed for $45 \mathrm{~min}$ to give a yellow precipitate. The solution was concentrated to a low volume and the yellow precipitate (4b) was filtered and washed with methanol, (22 mg, 88\%). Found: C, 69.62; H, 5.12; N 3.88, calcd. (\%) for $\mathrm{C}_{40} \mathrm{H}_{35} \mathrm{~N}_{2} \mathrm{ClPd}$ : C, 70.07; $\mathrm{H}, 5.15 ; \mathrm{N} 4.09$. IR (neat, $\mathrm{v}, \mathrm{cm}^{-1}$ ): 2949, 1546, 1582, 1422, 826 and 772. ESI-MS (MeCN, m/z): found: 649.1855; calcd. 649.1835 for $\mathrm{C}_{40} \mathrm{H}_{35} \mathrm{~N}_{2} \mathrm{Pd},[\mathrm{M}-\mathrm{Cl}]^{+} .{ }^{1} \mathrm{H}-\mathrm{NMR}(400 \mathrm{MHz}$, 
$\left.\mathrm{CDCl}_{3}\right)$ : $9.06\left(\mathrm{br}, \mathrm{m}, 1 \mathrm{H}, \mathrm{H}^{10}\right), 8.83\left(\mathrm{~d}, 1 \mathrm{H},{ }^{3} \mathrm{~J}(\mathrm{HH}) 7.5 \mathrm{~Hz}, \mathrm{H}^{3}\right), 8.24$ (d, $\left.1 \mathrm{H},{ }^{3} \mathrm{~J}(\mathrm{HH}) 8.0 \mathrm{~Hz}, \mathrm{H}^{13}\right), 8.05\left(\mathrm{~d}, 1 \mathrm{H},{ }^{4} \mathrm{~J}(\mathrm{HH}) 2.0 \mathrm{~Hz}, \mathrm{H}^{11}\right), 8.04(\mathrm{~d}, 1 \mathrm{H}$, $\left.3 \mathrm{~J}(\mathrm{HH}) 8.0 \mathrm{~Hz}, \mathrm{H}^{1}\right), 8.02\left(\mathrm{~d}, 1 \mathrm{H}, 3 \mathrm{~J}(\mathrm{HH}) 7.5 \mathrm{~Hz}, \mathrm{H}^{1}\right), 7.78$ (t, $1 \mathrm{H}, 3 \mathrm{~J}(\mathrm{HH})$ $\left.7.5 \mathrm{~Hz}, \mathrm{H}^{2}\right), 7.76\left(\mathrm{~d}, 2 \mathrm{H},{ }^{3} \mathrm{~J}(\mathrm{HH}) 8.0 \mathrm{~Hz}, \mathrm{H}^{5}\right), 7.51$ (dt, $1 \mathrm{H}, 3 \mathrm{~J}(\mathrm{HH}) 8.0$ $\left.\mathrm{Hz},{ }^{4} \mathrm{~J}(\mathrm{HH}) 1.5 \mathrm{~Hz}, \mathrm{H}^{8}\right), 7.44\left(\mathrm{~d}, 2 \mathrm{H}, 3 \mathrm{~J}(\mathrm{HH}) 8.0 \mathrm{~Hz}, \mathrm{H}^{4}\right), 7.39\left(\mathrm{~m}, 2 \mathrm{H}, \mathrm{H}^{2}\right.$ $\left.\& \mathrm{H}^{9}\right), 7.26\left(\mathrm{dd}, 1 \mathrm{H}, 3 \mathrm{~J}(\mathrm{HH}) 8.0 \mathrm{~Hz},{ }^{4} \mathrm{~J}(\mathrm{HH}) 2.0 \mathrm{~Hz}, \mathrm{H}^{12}\right), 6.84(\mathrm{~d}, 1 \mathrm{H}$, $\left.{ }^{3} \mathrm{~J}(\mathrm{HH}) 8.5 \mathrm{~Hz}, \mathrm{H}^{7}\right), 6.48$ (d, 1H, $\left.3 \mathrm{~J}(\mathrm{HH}) 7.5 \mathrm{~Hz}, \mathrm{H}^{3}\right), 1.54$ (s, 9H, $\left.\mathrm{CMe}_{3}\right)$ and $1.45\left(\mathrm{~s}, 9 \mathrm{H}, \mathrm{CMe}_{3}\right) .{ }^{13} \mathrm{C}-\mathrm{NMR}\left(100 \mathrm{MHz} \mathrm{CDCl}_{3}\right): 150.0\left(1 \mathrm{C}, \mathrm{C}^{10}\right)$, 133.8 (1C, $\left.\mathrm{C}^{11}\right), 138.1$ (1C, $\left.\mathrm{C}^{8}\right), 130.1$ (1C, $\left.\mathrm{C}^{1}\right), 128.9\left(1 \mathrm{C}, \mathrm{C}^{1}\right), 128.8$ (2C, C4), $128.1\left(1 \mathrm{C}, \mathrm{C}^{2}\right), 128.1\left(1 \mathrm{C}, \mathrm{C}^{2}\right), 127.6$ (2C, $\left.\mathrm{C}^{5}\right), 126.3$ (1C, C13), $126.7\left(1 \mathrm{C}^{3} \mathrm{C}^{3}\right), 126.2\left(1 \mathrm{C}, \mathrm{C}^{3}\right), 125.6\left(1 \mathrm{C}, \mathrm{C}^{7}\right), 125.4\left(1 \mathrm{C}, \mathrm{C}^{9}\right)$ and 121.2 $\left(1 \mathrm{C}, \mathrm{C}^{12}\right)$.

\section{[PdCl(L)] (4b) from (4a)}

A solution of $\mathrm{NH}_{4} \mathrm{Cl}(6 \mathrm{mg}, 0.112 \mathrm{mmol})$ in methanol $(1 \mathrm{~mL})$ was added to a suspension of (4a) $(15 \mathrm{mg}, 0.021 \mathrm{mmol})$ in acetone $(4 \mathrm{~mL})$ and dichloromethane $(1 \mathrm{~mL})$. The reaction mixture was stirred at room temperature for $15 \mathrm{~h}$; the solvent was then removed and the product extracted into dichloromethane. The combined extract was concentrated and methanol was added to give the required product (4b) as a yellow solid, (12 mg, 83\%).

\section{$[\operatorname{PdBr}(\mathrm{L})](\mathbf{4 c )}$ from (4a)}

A solution of $\mathrm{NaBr}(11 \mathrm{mg}, 0.109 \mathrm{mmol})$ in methanol $(1 \mathrm{~mL})$ was added to a suspension of (4a) (13 $\mathrm{mg}, 0.0183 \mathrm{mmol})$ in acetone $(4 \mathrm{~mL})$ and dichloromethane $(1 \mathrm{~mL})$. The reaction mixture was stirred at room temperature for $24 \mathrm{~h}$; the solvent was then removed and the residue was extracted with dichloromethane. The combined extract was concentrated and methanol was added to give the required product (4c) as a yellow solid, (11 mg, 83\%). Found: C, 65.55; H, 4.80; N 3.57, calcd. (\%) for $\mathrm{C}_{40} \mathrm{H}_{35} \mathrm{~N}_{2} \mathrm{BrPd}$ : C, 65.81; $\mathrm{H}, 4.83$; N 3.84. IR (neat, $v$, $\left.\mathrm{cm}^{-1}\right): 2960,1582,1545,1421,1276,1261,824,765$ and 750 . ESI-MS (acetonitrile, $\mathrm{m} / \mathrm{z}$ ): found: 649.1823; calcd. 649.1835 for $\mathrm{C}_{40} \mathrm{H}_{35} \mathrm{~N}_{2} \mathrm{Pd}$, $[\mathrm{M}-\mathrm{Br}]^{+} .{ }^{1} \mathrm{H}-\mathrm{NMR}\left(400 \mathrm{MHz}, \mathrm{CDCl}_{3}, \delta\right.$ in ppm): 9.21 (dd, $1 \mathrm{H},{ }^{3} \mathrm{~J}(\mathrm{HH}) 5.0$ $\left.\mathrm{Hz},{ }^{4} \mathrm{~J}(\mathrm{HH}) 2.0 \mathrm{~Hz}, \mathrm{H}^{10}\right), 8.81\left(\mathrm{~d}, 1 \mathrm{H},{ }^{3} \mathrm{~J}(\mathrm{HH}) 7.0 \mathrm{~Hz}, \mathrm{H}^{3}\right), 8.28(\mathrm{~d}, 1 \mathrm{H}$, $\left.{ }^{4} \mathrm{~J}(\mathrm{HH}) 2.0 \mathrm{~Hz}, \mathrm{H}^{11}\right), 8.23\left(\mathrm{~d}, 1 \mathrm{H},{ }^{3} \mathrm{~J}(\mathrm{HH}) 8.0 \mathrm{~Hz}, \mathrm{H}^{13}\right), 8.03(\mathrm{~d}, 1 \mathrm{H}$, $\left.{ }^{3} \mathrm{~J}(\mathrm{HH}) 8.0 \mathrm{~Hz}, \mathrm{H}^{1}\right), 8.01\left(\mathrm{~d}, 1 \mathrm{H},{ }^{3} \mathrm{~J}(\mathrm{HH}) 8.0 \mathrm{~Hz}, \mathrm{H}^{1}\right), 7.78\left(\mathrm{t}, 1 \mathrm{H},{ }^{3} \mathrm{~J}(\mathrm{HH})\right.$ $\left.8.0 \mathrm{~Hz}, \mathrm{H}^{2}\right), 7.76\left(\mathrm{~d}, 2 \mathrm{H},{ }^{3} \mathrm{~J}(\mathrm{HH}) 8.5 \mathrm{~Hz}, \mathrm{H}^{5}\right), 7.49$ (dt, $1 \mathrm{H},{ }^{3} \mathrm{~J}(\mathrm{HH}) 8.0$ $\left.\mathrm{Hz},{ }^{4} \mathrm{~J}(\mathrm{HH}) 1.5 \mathrm{~Hz}, \mathrm{H}^{8}\right), 7.43\left(\mathrm{~d}, 2 \mathrm{H},{ }^{3} \mathrm{~J}(\mathrm{HH}) 8.5 \mathrm{~Hz}, \mathrm{H}^{4}\right), 7.42\left(\mathrm{~m}, 1 \mathrm{H}, \mathrm{H}^{2}\right.$ overlap with $\left.\mathrm{H}^{4}\right), 7.35\left(\mathrm{~m}, 1 \mathrm{H},{ }^{3} \mathrm{~J}(\mathrm{HH}) 5.0 \mathrm{~Hz},{ }^{4} \mathrm{~J}(\mathrm{HH}) 1.5 \mathrm{~Hz}, \mathrm{H}^{9}\right), 7.25$ (dd, $\left.1 \mathrm{H},{ }^{3} \mathrm{~J}(\mathrm{HH}) 8.5 \mathrm{~Hz},{ }^{4} \mathrm{~J}(\mathrm{HH}) 2.0 \mathrm{~Hz}, \mathrm{H}^{12}\right), 6.85$ (d, $1 \mathrm{H},{ }^{3} \mathrm{~J}(\mathrm{HH}) 8.5 \mathrm{~Hz}$, $\left.\mathrm{H}^{7}\right), 6.48\left(\mathrm{~d}, 1 \mathrm{H},{ }^{3} \mathrm{~J}(\mathrm{HH}) 7.5 \mathrm{~Hz}, \mathrm{H}^{3}\right), 1.54\left(\mathrm{~s}, 9 \mathrm{H}, \mathrm{CMe}_{3}\right)$ and $1.44(\mathrm{~s}$, 
9H, $\left.\mathrm{CMe}_{3}\right) .{ }^{13} \mathrm{C}-\mathrm{NMR}\left(100 \mathrm{MHz}, \mathrm{CDCl}_{3}\right): 151.1$ (1C, C10), 136.4 (1C, $\left.\mathrm{C}^{11}\right)$, $137.9\left(1 \mathrm{C}, \mathrm{C}^{8}\right), 130.1\left(1 \mathrm{C}, \mathrm{C}^{1}\right), 129.0\left(1 \mathrm{C}, \mathrm{C}^{1}\right), 128.8\left(2 \mathrm{C}, \mathrm{C}^{4}\right), 128.0$ $\left(1 \mathrm{C}, \mathrm{C}^{2}\right), 128.1\left(1 \mathrm{C}, \mathrm{C}^{2}\right), 127.6\left(2 \mathrm{C}, \mathrm{C}^{5}\right), 126.4\left(1 \mathrm{C}, \mathrm{C}^{13}\right), 126.7\left(1 \mathrm{C}, \mathrm{C}^{3}\right)$, $126.2\left(1 \mathrm{C}, \mathrm{C}^{3}\right), 125.7\left(1 \mathrm{C}, \mathrm{C}^{7}\right), 125.6\left(1 \mathrm{C}, \mathrm{C}^{9}\right)$ and $121.0\left(1 \mathrm{C}, \mathrm{C}^{12}\right)$.

\section{[PdBr(L)] (4c) from (4b)}

A solution of $\mathrm{NaBr}(11 \mathrm{mg}, 0.109 \mathrm{mmol})$ in methanol $(1 \mathrm{~mL})$ was added to a suspension of (4b) (13 $\mathrm{mg}, 0.0189 \mathrm{mmol})$ in acetone $(4 \mathrm{~mL})$ and $\mathrm{CH}_{2} \mathrm{Cl}_{2}(1 \mathrm{~mL})$. The reaction mixture was stirred at room temperature for $20 \mathrm{~h}$; the solvent was then removed and the residue was extracted with dichloromethane. The combined extract was concentrated and methanol was added to give the required product $(\mathbf{4 c})$ as a yellow solid, (13 mg, 94\%).

\section{$[\operatorname{PdI}(L)](4 d)$}

To a suspension of (4a) $(15 \mathrm{mg}, 0.021 \mathrm{mmol})$ in acetone $(4 \mathrm{~mL})$ and $\mathrm{CH}_{2} \mathrm{Cl}_{2}(1 \mathrm{~mL})$ was added $\mathrm{NaI}(16 \mathrm{mg}, 0.105 \mathrm{mmol})$. The reaction mixture was stirred at room temperature for $20 \mathrm{~h}$; the solvent was then removed and the residue extracted with $\mathrm{CH}_{2} \mathrm{Cl}_{2}$. The combined extract was concentrated and added methanol to give the required product (4d) as a yellow solid, (15 mg, 91\%). Found: C, 61.46; H, 4.53; N 3.34, calcd. (\%) for $\mathrm{C}_{40} \mathrm{H}_{35} \mathrm{~N}_{2} \mathrm{IPd}$ : C, 61.83; H, 4.54; N 3.61. IR (neat, $\mathrm{v}, \mathrm{cm}^{-1}$ ): 2960, 1584, 1547, 1420, 824 and 771. ESI-MS (acetonitrile, $\mathrm{m} / \mathrm{z}$ ):

found: 649.1865; calcd. 649.1835 for $\mathrm{C}_{40} \mathrm{H}_{35} \mathrm{~N}_{2} \mathrm{Pd}$, [M-I] ${ }^{+}$. ${ }^{1} \mathrm{H}-\mathrm{NMR}(400$ $\mathrm{MHz}, \mathrm{CDCl}_{3}, \delta$ in ppm): $9.45\left(\mathrm{dd}, 1 \mathrm{H},{ }^{3} \mathrm{~J}(\mathrm{HH}) 5.0 \mathrm{~Hz},{ }^{4} \mathrm{~J}(\mathrm{HH}) 1.0 \mathrm{~Hz}\right.$, $\left.\mathrm{H}^{10}\right), 8.80\left(\mathrm{~d}, 1 \mathrm{H},{ }^{3} \mathrm{~J}(\mathrm{HH}) 7.0 \mathrm{~Hz}, \mathrm{H}^{3}\right), 8.65\left(\mathrm{~d}, 1 \mathrm{H},{ }^{4} \mathrm{~J}(\mathrm{HH}) 2.0 \mathrm{~Hz}, \mathrm{H}^{11}\right)$, $8.23\left(\mathrm{~d}, 1 \mathrm{H},{ }^{3} \mathrm{~J}(\mathrm{HH}) 8.0 \mathrm{~Hz}, \mathrm{H}^{13}\right), 8.04\left(\mathrm{~d}, 1 \mathrm{H},{ }^{3} \mathrm{~J}(\mathrm{HH}) 8.0 \mathrm{~Hz}, \mathrm{H}^{1}\right), 8.02$ $\left(\mathrm{d}, 1 \mathrm{H}, 3 \mathrm{~J}(\mathrm{HH}) 8.0 \mathrm{~Hz}, \mathrm{H}^{1}\right), 7.76\left(\mathrm{t}, 1 \mathrm{H}, 3 \mathrm{~J}(\mathrm{HH}) 8.0 \mathrm{~Hz}, \mathrm{H}^{2}\right), 7.75$ (d, 2H, $\left.{ }^{3} \mathrm{~J}(\mathrm{HH}) 8.5 \mathrm{~Hz}, \mathrm{H}^{5}\right), 7.48\left(\mathrm{dt}, 1 \mathrm{H}, 3 \mathrm{~J}(\mathrm{HH}) 8.0 \mathrm{~Hz},{ }^{4} \mathrm{~J}(\mathrm{HH}) 1.5 \mathrm{~Hz}, \mathrm{H}^{8}\right), 7.42$ $\left(\mathrm{d}, 2 \mathrm{H},{ }^{3} \mathrm{~J}(\mathrm{HH}) 8.5 \mathrm{~Hz}, \mathrm{H}^{4}\right), 7.42\left(\mathrm{~m}, 1 \mathrm{H}, \mathrm{H}^{2}\right.$, overlap with $\left.\mathrm{H}^{4}\right), 7.33(\mathrm{~m}$, $\left.1 \mathrm{H},{ }^{3} \mathrm{~J}(\mathrm{HH}) 7.5 \mathrm{~Hz},{ }^{4} \mathrm{~J}(\mathrm{HH}) 1.0 \mathrm{~Hz}, \mathrm{H}^{9}\right), 7.23\left(\mathrm{dd}, 1 \mathrm{H},{ }^{3} \mathrm{~J}(\mathrm{HH}) 8.0 \mathrm{~Hz}\right.$, $\left.{ }^{4} \mathrm{~J}(\mathrm{HH}) 2.0 \mathrm{~Hz}, \mathrm{H}^{12}\right), 6.88\left(\mathrm{~d}, 1 \mathrm{H},{ }^{3} \mathrm{~J}(\mathrm{HH}) 8.0 \mathrm{~Hz}, \mathrm{H}^{7}\right), 6.48\left(\mathrm{~d}, 1 \mathrm{H},{ }^{3} \mathrm{~J}(\mathrm{HH})\right.$ $\left.7.0 \mathrm{~Hz}, \mathrm{H}^{3}\right), 1.54\left(\mathrm{~s}, 9 \mathrm{H}, \mathrm{CMe}_{3}\right)$ and $1.43\left(\mathrm{~s}, 9 \mathrm{H}, \mathrm{CMe}_{3}\right) .{ }^{13} \mathrm{C}-\mathrm{NMR}(100$ $\mathrm{MHz}, \mathrm{CDCl}_{3}, \delta$ in ppm): $153.4\left(1 \mathrm{C}, \mathrm{C}^{10}\right), 141.4\left(1 \mathrm{C}, \mathrm{C}^{11}\right), 137.5\left(1 \mathrm{C}, \mathrm{C}^{8}\right)$, 130.1 (1C, C1), 129.0 (1C, $\left.{ }^{1}\right), 128.8\left(2 \mathrm{C}, \mathrm{C}^{4}\right), 128.1\left(1 \mathrm{C}, \mathrm{C}^{2}\right), 128.1$ $\left(1 \mathrm{C}, \mathrm{C}^{2}\right), 127.6\left(2 \mathrm{C}, \mathrm{C}^{5}\right), 126.7\left(1 \mathrm{C}, \mathrm{C}^{13}\right), 126.7\left(1 \mathrm{C}, \mathrm{C}^{3}\right), 126.1\left(1 \mathrm{C}, \mathrm{C}^{3}\right)$, $125.9\left(1 \mathrm{C}, \mathrm{C}^{7}\right), 125.8\left(1 \mathrm{C}, \mathrm{C}^{9}\right)$ and $120.6\left(1 \mathrm{C}, \mathrm{C}^{12}\right)$.

\section{$\left[\left(\eta^{3}-\right.\right.$ methallyl)Pd(2b)]PF 6 (5)}

The ligand (2b) $(20 \mathrm{mg}, 0.037 \mathrm{mmol})$ and $\left[\left(\eta^{3}-\text { metallyl }\right) \operatorname{Pd}(\mu-\mathrm{Cl})\right]_{2}$ (7.2 $\mathrm{mg}, 0.018 \mathrm{mmol})$ were dissolved in dichloromethane $(1 \mathrm{~mL})$. After 
$15 \mathrm{~min}$, a solution of $\mathrm{NH}_{4} \mathrm{PF}_{6}(12 \mathrm{mg}, 0.073 \mathrm{mmol})$ in methanol $(1 \mathrm{~mL})$ was added. The solution was concentrated to yield the required product (5) as a yellow solid (26 mg, 81\%). Found: C, 61.31; H, 5.02; N 3.18, calcd. (\%) for $\mathrm{C}_{44} \mathrm{H}_{43} \mathrm{~N}_{2} \mathrm{PF}_{6} \mathrm{Pd} \cdot 0.2 \mathrm{CH}_{2} \mathrm{Cl}_{2}$ : C, 61.14; $\mathrm{H}, 5.04 ; \mathrm{N}$ 3.22. IR (neat, $v, \mathrm{~cm}^{-1}$ ): 2959, 1610, 1559, 1476, 1460, 1420, 1275, 1267, 1116 and 829. ESI-MS (acetone, m/z): found: 705.2438; calcd. 705.2461 for $\mathrm{C}_{44} \mathrm{H}_{43} \mathrm{~N}_{2} \mathrm{Pd},\left[\mathrm{M}-\mathrm{PF}_{6}\right]^{+}$. ${ }^{1} \mathrm{H}-\mathrm{NMR}\left(400 \mathrm{MHz}, \mathrm{CDCl}_{3}, \delta\right.$ in ppm): $8.92\left(\mathrm{br}, \mathrm{d}, 1 \mathrm{H},{ }^{3} \mathrm{~J}(\mathrm{HH}) 4.5 \mathrm{~Hz}, \mathrm{H}^{10}\right), 8.06\left(\mathrm{~d}, 1 \mathrm{H},{ }^{3} \mathrm{~J}(\mathrm{HH}) 8.0 \mathrm{~Hz}\right.$, $\mathrm{H}^{1}$ or $\left.\mathrm{H}^{1}\right), 7.99\left(\mathrm{~d}, 1 \mathrm{H},{ }^{3} \mathrm{~J}(\mathrm{HH}) 8.0 \mathrm{~Hz}, \mathrm{H}^{1}\right.$ or $\left.\mathrm{H}^{1}\right), 7.78-7.76\left(\mathrm{~m}, 6 \mathrm{H}, \mathrm{H}^{5}\right.$, $\mathrm{H}^{4}$ and $\left.\mathrm{H}^{5}\right), 7.62-7.54\left(\mathrm{~m}, 4 \mathrm{H}, \mathrm{H}^{2}, \mathrm{H}^{2}, \mathrm{H}^{8}\right.$ and $\left.\mathrm{H}^{9}\right), 7.52\left(\mathrm{~d}, 2 \mathrm{H},{ }^{3} \mathrm{~J}(\mathrm{HH})\right.$ $\left.8.0 \mathrm{~Hz}, \mathrm{H}^{4}\right), 7.18\left(\mathrm{~d}, 2 \mathrm{H},{ }^{3} \mathrm{~J}(\mathrm{HH}) 7.0 \mathrm{~Hz}, \mathrm{H}^{3}\right.$ and $\left.\mathrm{H}^{3}\right), 6.89\left(\mathrm{~d}, 1 \mathrm{H},{ }^{3} \mathrm{~J}(\mathrm{HH})\right.$ $7.0 \mathrm{~Hz}, \mathrm{H}^{7}$ ), 3.99 (br, 1H, allyl-H), 3.30 (br, 1H, allyl-H), 2.48 (br, $1 \mathrm{H}$, allyl-H), 2.01 (s, 3H, Me), 1.85 (br, 1H, allyl-H), 1.51 (s, 9H, $\left.\mathrm{CMe}_{3}\right)$ and $1.53\left(\mathrm{~s}, 9 \mathrm{H}, \mathrm{CMe}_{3}\right) .{ }^{13} \mathrm{C}-\mathrm{NMR}\left(100 \mathrm{MHz}, \mathrm{CDCl}_{3}, \delta\right.$ in ppm): $153.9(1 \mathrm{C}$, $\left.\mathrm{C}^{10}\right), 138.1$ (1C, $\left.\mathrm{C}^{8}\right), 130.6$ (1C, $\mathrm{C}^{1}$ or $\mathrm{C}^{1}$ ), 129.3 ( $\left.\mathrm{C}_{\text {quat }}\right), 129.1$ (1C, $\mathrm{C}^{1}$ or $\mathrm{C}^{1}$ ), 128.9 (Ar), 128.4 (Ar), 128.1 ( $\mathrm{C}^{2}$ or $\mathrm{C}^{2}$ ), 127.9 (1C, $\mathrm{C}^{2}$ or $\left.\mathrm{C}^{2}\right), 127.5$ (1C, $\mathrm{C}^{7}$ ), 127.1 (Ar), $126.2\left(1 \mathrm{C}, \mathrm{C}^{3}\right.$ or $\left.\mathrm{C}^{3}\right), 125.7$ (Ar), 125.5 (1C, $\mathrm{C}^{3}$ or $\mathrm{C}^{3}$ ), 125.4 (1C, $\left.\mathrm{C}^{9}\right), 34.8$ (1C, $\left.\mathrm{CMe}_{3}\right), 34.7$ (1C, $\left.\mathrm{CMe}_{3}\right), 31.0\left(3 \mathrm{C}, \mathrm{CMe}_{3}\right)$, $31.0\left(3 \mathrm{C}, \mathrm{CMe}_{3}\right)$ and $22.1(1 \mathrm{C}, \mathrm{Me})$.

\section{$[P d(D M A P)(L)] P F_{6}(6 a)$}

To a suspension of (4a) (14 mg, $0.0197 \mathrm{mmol})$ in $\mathrm{CH}_{2} \mathrm{Cl}_{2}(2 \mathrm{~mL})$ was added 4-dimethylaminopyridine (DMAP) $(10 \mathrm{mg}, 0.082 \mathrm{mmol})$ followed by $\mathrm{NH}_{4} \mathrm{PF}_{6}(12 \mathrm{mg}, 0.073 \mathrm{mmol})$ in methanol $(1 \mathrm{~mL})$. After $15 \mathrm{~min}$, the resulting pale yellow solution was concentrated to give (6a) as a yellow solid, (16 mg, 89\%). Found: C, 60.29; H, 4.82; N 5.90, calcd. (\%) for $\mathrm{C}_{47} \mathrm{H}_{45} \mathrm{~N}_{4} \mathrm{PF}_{6} \mathrm{Pd} \cdot 0.25 \mathrm{CH}_{2} \mathrm{Cl}_{2}: \mathrm{C}, 60.47 ; \mathrm{H}, 4.89 ; \mathrm{N}$ 5.98. IR (neat, $\mathrm{v}, \mathrm{cm}^{-1}$ ): 2948, 1618, 1541, 1422, 1392, 1275, 1261, 1224, 835, 750 and 764 .

ESI-MS (acetone, $\mathrm{m} / \mathrm{z}$ ): found: 771.2663 ; calcd. 771.2679 for $\mathrm{C}_{47} \mathrm{H}_{45} \mathrm{~N}_{4} \mathrm{Pd},\left[\mathrm{M}-\mathrm{PF}_{6}\right]^{+} .{ }^{1} \mathrm{H}-\mathrm{NMR}\left(600 \mathrm{MHz}, \mathrm{CDCl}_{3}, \delta\right.$ in ppm): $8.81(\mathrm{~d}, 1 \mathrm{H}$, $\left.{ }^{3} \mathrm{~J}(\mathrm{HH}) 7.5 \mathrm{~Hz}, \mathrm{H}^{3}\right), 8.40\left(\mathrm{~d}, 2 \mathrm{H},{ }^{3} \mathrm{~J}(\mathrm{HH}) 7.0 \mathrm{~Hz}, \mathrm{H}^{2}\right.$ of DMAP), 8.35 (d, $\left.1 \mathrm{H},{ }^{3} \mathrm{~J}(\mathrm{HH}) 8.0 \mathrm{~Hz}, \mathrm{H}^{13}\right), 8.09\left(\mathrm{~d}, 1 \mathrm{H},{ }^{3} \mathrm{~J}(\mathrm{HH}) 8.0 \mathrm{~Hz}, \mathrm{H}^{1}\right), 8.06(\mathrm{~d}, 1 \mathrm{H}$, $\left.{ }^{3} \mathrm{~J}(\mathrm{HH}) 8.0 \mathrm{~Hz}, \mathrm{H}^{1}\right), 8.04\left(\mathrm{br}, \mathrm{d}, 1 \mathrm{H},{ }^{3} \mathrm{~J}(\mathrm{HH}) 6.5 \mathrm{~Hz}, \mathrm{H}^{10}\right.$, overlaps with $\left.\mathrm{H}^{4}\right), 7.82\left(\mathrm{t}, 1 \mathrm{H},{ }^{3} \mathrm{~J}(\mathrm{HH}) 8.0 \mathrm{~Hz}, \mathrm{H}^{2}\right), 7.79\left(\mathrm{~d}, 2 \mathrm{H},{ }^{3} \mathrm{~J}(\mathrm{HH}) 8.5 \mathrm{~Hz}, \mathrm{H}^{5}\right)$, $7.65\left(\mathrm{~m}, 1 \mathrm{H},{ }^{3} \mathrm{~J}(\mathrm{HH}) 6.5 \mathrm{~Hz}, \mathrm{H}^{9}\right), 7.59\left(\mathrm{~m}, 1 \mathrm{H},{ }^{3} \mathrm{~J}(\mathrm{HH}) 8.0 \mathrm{~Hz},{ }^{4} \mathrm{~J}(\mathrm{HH}) 1.5\right.$ $\left.\mathrm{Hz}, \mathrm{H}^{8}\right), 7.42\left(\mathrm{~m}, 1 \mathrm{H},{ }^{3} \mathrm{~J}(\mathrm{HH}) 8.0 \mathrm{~Hz}, \mathrm{H}^{2}\right.$ overlaps with $\left.\mathrm{H}^{4}\right), 7.43(\mathrm{~d}, 2 \mathrm{H}$, $\left.{ }^{3} \mathrm{~J}(\mathrm{HH}) 8.5 \mathrm{~Hz}, \mathrm{H}^{4}\right), 7.34\left(\mathrm{dd}, 1 \mathrm{H},{ }^{3} \mathrm{~J}(\mathrm{HH}) 8.5 \mathrm{~Hz},{ }^{4} \mathrm{~J}(\mathrm{HH}) 2.0 \mathrm{~Hz}, \mathrm{H}^{12}\right)$, $6.92\left(\mathrm{~d}, 1 \mathrm{H}, 3 \mathrm{~J}(\mathrm{HH}) 8.5 \mathrm{~Hz}, \mathrm{H}^{7}\right), 6.84\left(\mathrm{~d}, 2 \mathrm{H}, 3 \mathrm{~J}(\mathrm{HH}) 7.0 \mathrm{~Hz}, \mathrm{H}^{3}\right.$ of DMAP), 6.82 (d, 1H, $\left.{ }^{4} \mathrm{~J}(\mathrm{HH}) 2.0 \mathrm{~Hz}, \mathrm{H}^{11}\right), 6.53$ (d, 1H, $3 \mathrm{~J}(\mathrm{HH}) 7.0 \mathrm{~Hz}$, $\left.\mathrm{H}^{3}\right), 3.23\left(\mathrm{~s}, 6 \mathrm{H}, \mathrm{NMe}_{2}\right), 1.55\left(\mathrm{~s}, 9 \mathrm{H}, \mathrm{CMe}_{3}\right)$ and $1.32\left(\mathrm{~s}, 9 \mathrm{H}, \mathrm{CMe}_{3}\right) .{ }^{13} \mathrm{C}-$ NMR (150.9 MHz, $\mathrm{CDCl}_{3}, \delta$ in ppm): 150.4 (1C, C10), 139.3 (1C, C8), $131.1\left(1 \mathrm{C}, \mathrm{C}^{11}\right), 130.6\left(1 \mathrm{C}, \mathrm{C}^{1}\right), 129.4\left(1 \mathrm{C}, \mathrm{C}^{1}\right), 128.6\left(2 \mathrm{C}, \mathrm{C}^{4}\right), 128.3$ 
Synthesis and coordination chemistry of $N$-doped polyphenylenes

(1C, $\left.\mathrm{C}^{2}\right), 128.1\left(1 \mathrm{C}, \mathrm{C}^{2}\right), 127.8$ (2C, $\left.\mathrm{C}^{5}\right), 127.7$ (1C, C9), $127.4\left(1 \mathrm{C}, \mathrm{C}^{7}\right)$, $127.3\left(1 \mathrm{C}, \mathrm{C}^{3}\right), 126.9\left(1 \mathrm{C}, \mathrm{C}^{13}\right), 126.2\left(1 \mathrm{C}, \mathrm{C}^{3}\right)$ and $122.1\left(1 \mathrm{C}, \mathrm{C}^{12}\right)$.

\section{$\left[P d\left(P P h_{3}\right)(L)\right] P F_{6}(6 b)$}

To a solution of (4a) $(15 \mathrm{mg}, 0.021 \mathrm{mmol})$ in $\mathrm{CH}_{2} \mathrm{Cl}_{2}(3 \mathrm{~mL})$ was added $\mathrm{PPh}_{3}(7 \mathrm{mg}, 0.026 \mathrm{mmol})$ followed by $\mathrm{NH}_{4} \mathrm{PF}_{6}(12 \mathrm{mg}, 0.073 \mathrm{mmol})$ in methanol $(1 \mathrm{~mL})$. After $30 \mathrm{~min}$, the resulting pale yellow solution was concentrated to give (6b) as a yellow solid (19 $\mathrm{mg}, 85 \%)$. Found: C, 62.25; H, 4.73; N 2.46, calcd. (\%) for $\mathrm{C}_{58} \mathrm{H}_{50} \mathrm{~N}_{2} \mathrm{P}_{2} \mathrm{~F}_{6} \mathrm{Pd}$ : C, 65.88; $\mathrm{H}$, 4.77; N 2.65. IR (neat, $v, \mathrm{~cm}^{-1}$ ): 2966, 1584, 1419, 1276, 1261, 834, 765 and 750. ESI-MS (acetone, $\mathrm{m} / \mathrm{z}$ ): found: 911.2779 ; calcd.

911.2746 for $\mathrm{C}_{58} \mathrm{H}_{50} \mathrm{~N}_{2} \mathrm{PPd},\left[\mathrm{M}-\mathrm{PF}_{6}\right]^{+}$: ${ }^{31} \mathrm{P}-\mathrm{NMR}\left(161 \mathrm{MHz}, \mathrm{CDCl}_{3}, \delta\right.$ in ppm): $42.4\left(\mathrm{~s}, \mathrm{PPh}_{3}\right)$ and -143.2 (septet, $\left.\mathrm{PF}_{6}{ }^{-}\right) .{ }^{1} \mathrm{H}-\mathrm{NMR}(600 \mathrm{MHz}$, $\mathrm{CDCl}_{3}, \delta$ in ppm): $8.89\left(\mathrm{~d}, 1 \mathrm{H},{ }^{3} \mathrm{~J}(\mathrm{HH}) 7.0 \mathrm{~Hz}, \mathrm{H}^{3}\right), 8.35\left(\mathrm{~d}, 1 \mathrm{H},{ }^{3} \mathrm{~J}(\mathrm{HH})\right.$ $\left.8.0 \mathrm{~Hz}, \mathrm{H}^{13}\right), 8.13\left(\mathrm{~d}, 1 \mathrm{H}, 3 \mathrm{~J}(\mathrm{HH}) 8.0 \mathrm{~Hz}, \mathrm{H}^{1}\right), 8.10(\mathrm{~d}, 1 \mathrm{H}, 3 \mathrm{~J}(\mathrm{HH}) 8.0$ $\left.\mathrm{Hz}, \mathrm{H}^{1}\right), 7.88-7.84\left(\mathrm{~m}, 6 \mathrm{H}, \mathrm{Ph}\right.$ and $\left.\mathrm{H}^{2}\right), 7.79\left(\mathrm{~d}, 2 \mathrm{H},{ }^{3} \mathrm{~J}(\mathrm{HH}) 8.5 \mathrm{~Hz}, \mathrm{H}^{5}\right)$, $7.64-7.54\left(\mathrm{~m}, 11 \mathrm{H}, \mathrm{Ph}\right.$ and $\left.\mathrm{H}^{8}\right), 7.49\left(\mathrm{t}, 1 \mathrm{H},{ }^{3} \mathrm{~J}(\mathrm{HH}) 8.0 \mathrm{~Hz}, \mathrm{H}^{2}\right), 7.46(\mathrm{~d}$, $\left.2 \mathrm{H},{ }^{3} \mathrm{~J}(\mathrm{HH}) 8.5 \mathrm{~Hz}, \mathrm{H}^{4}\right), 7.25\left(\mathrm{dd}, 1 \mathrm{H},{ }^{3} \mathrm{~J}(\mathrm{HH}) 8.0 \mathrm{~Hz},{ }^{4} \mathrm{~J}(\mathrm{HH}) 2.0 \mathrm{~Hz}\right.$, $\left.\mathrm{H}^{12}\right), 7.05\left(\mathrm{~m}, 2 \mathrm{H}, \mathrm{H}^{7}\right.$ and $\left.\mathrm{H}^{9}\right), 6.75\left(\mathrm{dd}, 1 \mathrm{H},{ }^{4} \mathrm{~J}(\mathrm{PH}) 6.0 \mathrm{~Hz},{ }^{4} \mathrm{~J}(\mathrm{HH}) 2.0\right.$ $\left.\mathrm{Hz}, \mathrm{H}^{11}\right), 6.63$ (br, d, 1H, 3J(HH) $\left.6.5 \mathrm{~Hz}, \mathrm{H}^{10}\right), 6.57$ (d, 1H, 3J(HH) 7.0 $\left.\mathrm{Hz}, \mathrm{H}^{3}\right), 1.53\left(\mathrm{~s}, 9 \mathrm{H}, \mathrm{CMe}_{3}\right)$ and $0.82\left(\mathrm{~s}, 9 \mathrm{H}, \mathrm{CMe}_{3}\right) .{ }^{13} \mathrm{C}-\mathrm{NMR}(150.9$ $\mathrm{MHz} \mathrm{CDCl}_{3}, \delta$ in ppm): $150.8\left(1 \mathrm{C}, \mathrm{C}^{10}\right), 139.7\left(1 \mathrm{C}, \mathrm{C}^{8}\right), 137.2\left(1 \mathrm{C}, \mathrm{C}^{11}\right)$, $130.9\left(1 \mathrm{C}, \mathrm{C}^{1}\right), 129.8\left(1 \mathrm{C}, \mathrm{C}^{1}\right), 128.6\left(2 \mathrm{C}, \mathrm{C}^{4}\right), 128.5\left(1 \mathrm{C}, \mathrm{C}^{2}\right), 128.1$ (1C, $\left.\mathrm{C}^{2}\right), 127.9$ (2C, C5), 127.7 (1C, C13), $127.7\left(1 \mathrm{C}, \mathrm{C}^{3}\right), 127.1$ (1C, $\left.\mathrm{C}^{7}\right)$, $126.3\left(1 \mathrm{C}, \mathrm{C}^{3}\right), 126.3\left(1 \mathrm{C}, \mathrm{C}^{9}\right), 121.9\left(1 \mathrm{C}, \mathrm{C}^{12}\right), 31.0\left(3 \mathrm{C}, \mathrm{CMe}_{3}\right)$, and $30.1\left(3 \mathrm{C}, \mathrm{CMe}_{3}\right)$.

\section{Results and Discussion}

\section{Ligands and molybdenum complexes}

The ligands (2a) and (2b) were prepared by a Diels-Alder cycloaddition reaction between 2 -cyanopyridine and the corresponding cyclopentadienone $(\mathbf{1} \mathbf{a})$ and $(\mathbf{1 b})$, respectively (Scheme 1). Characterising data for the ligands and other metal complexes are given in the experimental section. Spectroscopic data are discussed later. 


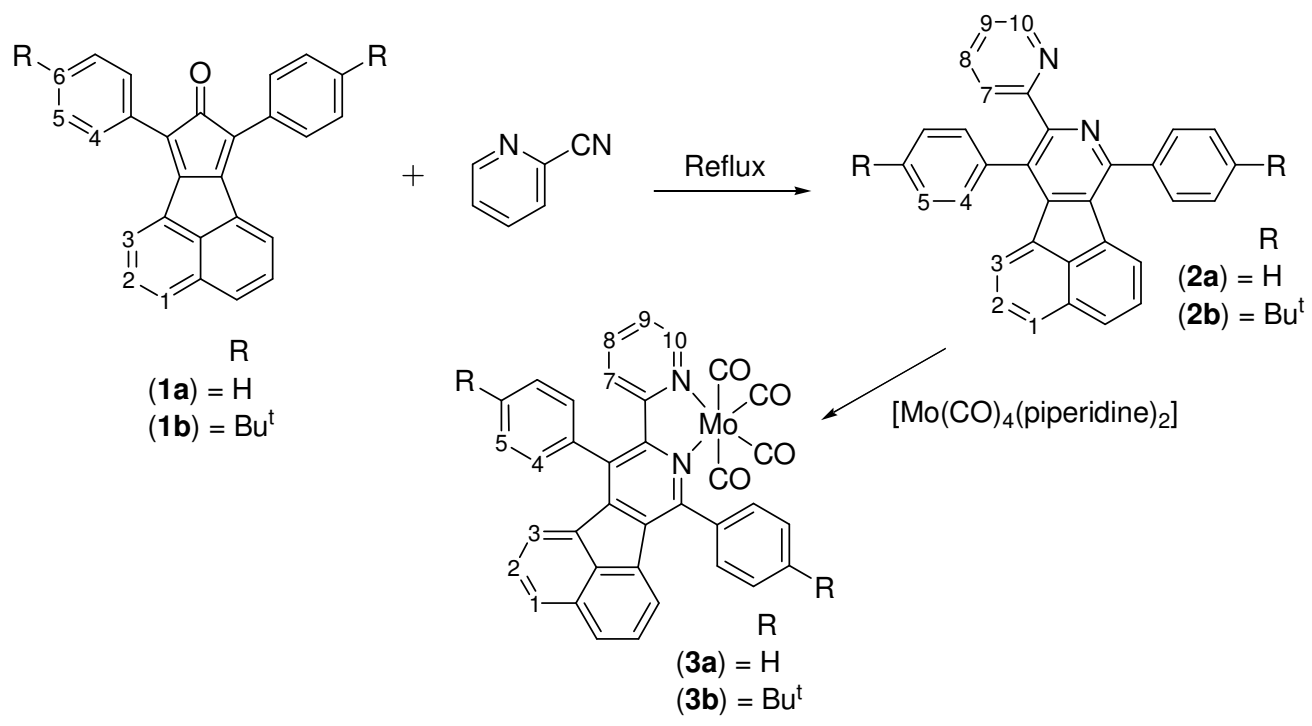

Scheme 1 Synthesis of ligands (2) and molybdenum complexes (3), and atom labelling used for the assignment of NMR data.

First we studied the coordination chemistry of these two ligands with zerovalent molybdenum centres stabilised by carbonyl ligands. Replacement of the two labile piperidine molecules of $\left[\mathrm{Mo}(\mathrm{CO})_{4}(\text { piperidine })_{2}\right]$ (Darensbourg \& Kump, 1978) with ligands (2) gave the tetracarbonylmolybdenum complexes of the type $\left[\mathrm{Mo}(\mathrm{CO})_{4}(2)\right]$ (3) in which the ligand is coordinated to the metal through both nitrogen donors.

\section{Palladium complexes}

The coordination chemistry of the ligand $(\mathbf{2} \mathbf{b})$ with palladium(II) centres was investigated as similar ligand systems are known to undergo cyclometallation via $\mathrm{C}-\mathrm{H}$ bond activation (Ollagnier et al 2008).

We decided to use the ligand (2b) $(\mathrm{LH})$ with tert-butyl groups at the para-positions due to easy identification of the cyclometallated complexes by NMR spectroscopy and to increase the solubility of the resulting palladium complexes. Treatment of $(\mathbf{2 b})$ with $\left[\mathrm{Pd}(\mathrm{OAc})_{2}\right]$ in refluxing dichloromethane resulted in the formation of the cyclometallated palladium(II) complex $[\mathrm{Pd}(\mathrm{OAc})(\mathrm{L})](\mathbf{4 a})$ as a yellow solid in $94 \%$ yield (Scheme 2). Treatment of $(\mathbf{2 b})$ with $\left[\mathrm{PdCl}_{2}\left(\mathrm{NCPh}_{2}\right]\right.$ in refluxing dichloromethane resulted in the formation of the palladium(II) complex $[\mathrm{PdCl}(\mathrm{L})](\mathbf{4 b})$ as a yellow solid in $88 \%$ yield. 
We studied the substitution reactions of $(\mathbf{4 a})$ and $(\mathbf{4 b})$ with monoanionic ligands such as halides. The acetate group of (4a) can be easily replaced by halide ions to give $[\mathrm{PdCl}(\mathrm{L})](\mathbf{4 b}),[\mathrm{PdBr}(\mathrm{L})](\mathbf{4} \mathbf{c})$ and $[\operatorname{PdI}(\mathrm{L})](\mathbf{4 d})$ respectively. Treatment of $(\mathbf{2 b})$ with 0.5 equivalent of $\left[\left(\eta^{3}-\right.\right.$ methallyl) $\operatorname{Pd}(\mu-\mathrm{Cl})]_{2}$ in dichloromethane and subsequent addition of $\mathrm{NH}_{4} \mathrm{PF}_{6}$ in methanol resulted in the formation of the $\mathrm{N}-\mathrm{N}$ chelate complex (5) as a yellow solid in $81 \%$ yield.

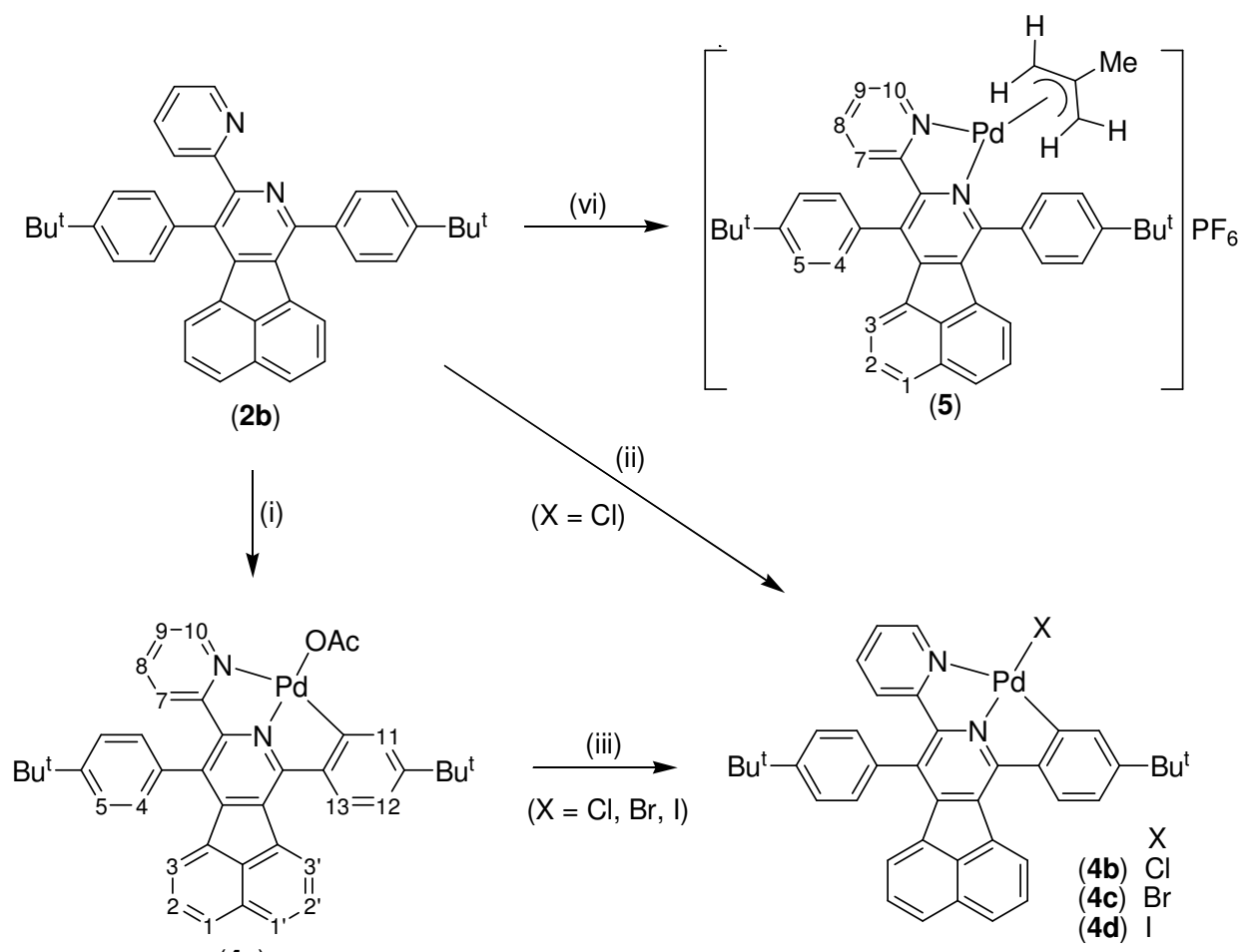

(4a)

Scheme 2 (i) $\left[\mathrm{Pd}(\mathrm{OAc})_{2}\right]$; (ii) $\left[\mathrm{PdCl}_{2}\left(\mathrm{NCPh}_{2}\right]\right.$; (iii) $\mathrm{NH}_{4} \mathrm{Cl}$ or $\mathrm{NaBr}$ or NaI; (vi) $\left[\left(\eta^{3}-\text { methallyl }\right) \mathrm{Pd}(\mu-\mathrm{Cl})\right]_{2}$.

We also studied the substitution reactions of (4a) with neutral ligands such as 4-dimethylaminopyridine (DMAP) and triphenylphosphine (Scheme 3). 

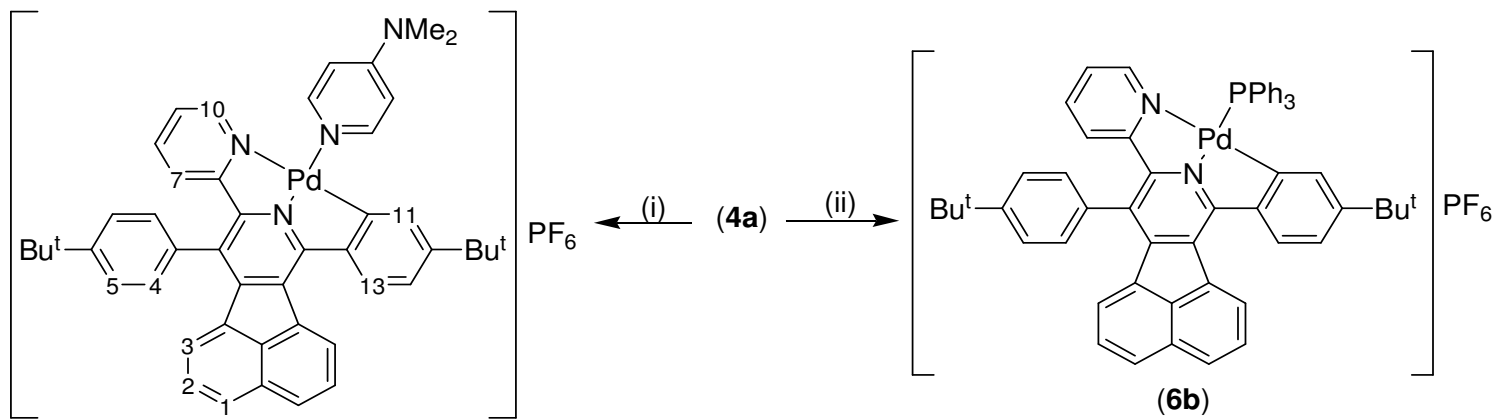

(6a)

(6b)

Scheme 3 (i) 4-Dimethylaminopyridine/ $\mathrm{NH}_{4} \mathrm{PF}_{6}$;

(ii) triphenylphosphine $/ \mathrm{NH}_{4} \mathrm{PF}_{6}$.

Treatment of (4a) with DMAP in dichloromethane and subsequent addition of $\mathrm{NH}_{4} \mathrm{PF}_{6}$ in methanol resulted in the formation of the salt (6a) as a yellow solid in $89 \%$ yield. The analogous phosphine complex (6b) was prepared similarly in $85 \%$ yield.

\section{Spectroscopic characterisation}

The compounds were fully characterized using IR, NMR, mass spectrometry and elemental analysis. For complexes (4a), (4b), (4c), $(\mathbf{4 d}),(5),(6 \mathbf{a})$ and $(\mathbf{6 b})$, the ESI-MS gave signals in agreement with the theoretically expected masses and isotopic distributions of $[\mathrm{M}-\mathrm{OAc}]^{+}$, $[\mathrm{M} \text {-halide }]^{+}$or $\left[\mathrm{M}-\mathrm{PF}_{6}\right]^{+}$. The ligands $(\mathbf{2 a})$ and $(\mathbf{2 b})$ gave an isotopic distribution of $[\mathrm{M}+1]^{+}$. Molybdenum complexes did not yield ESI-MS spectra. The ${ }^{1} \mathrm{H}$ and ${ }^{13} \mathrm{C}$ NMR chemical shifts were assigned by performing $\mathrm{H}-\mathrm{H}$ and $\mathrm{C}-\mathrm{H}$ COSY and NOE experiments.

The ${ }^{1} \mathrm{H}$ and ${ }^{13} \mathrm{C}$ NMR data observed for the 2-pyridyl group of the ligands $(\mathbf{2 a})$ and $(\mathbf{2 b}),(\delta) 7.5\left(\mathrm{H}^{7}\right), 7.6\left(\mathrm{H}^{8}\right), 7.12\left(\mathrm{H}^{9}\right), 8.52\left(\mathrm{H}^{10}\right), 124.6$ $\left(\mathrm{C}^{7}\right), 135\left(\mathrm{C}^{8}\right), 121.6\left(\mathrm{C}^{9}\right)$ and $148.4\left(\mathrm{C}^{10}\right) \mathrm{ppm}$, are in good agreement with the values reported in the literature (Ollagnier et al 2008 and Hii et al, 1995). The ${ }^{1} \mathrm{H}$ NMR spectrum of $(\mathbf{2} \mathbf{b})$ displays two sets of ABpatterns with ${ }^{3} \mathrm{~J}(\mathrm{HH})=8.5 \mathrm{~Hz}$, consistent with the presence of two aryl groups.

The molybdenum complexes (3a) and $(\mathbf{3 b})$ were fully characterized. The IR spectrum of (3a) showed three IR bands at 2005, 1863 and 1823 $\mathrm{cm}^{-1}$ for the carbonyl ligands. The aromatic region of the ${ }^{1} \mathrm{H}$ NMR spectrum of $(\mathbf{3 b})$ is shown in Figure 3. 
Figure 3 Aromatic region of the ${ }^{1} \mathrm{H}$ NMR spectrum of $(\mathbf{3 b})$ with the atom labelling used for the assignment of NMR data

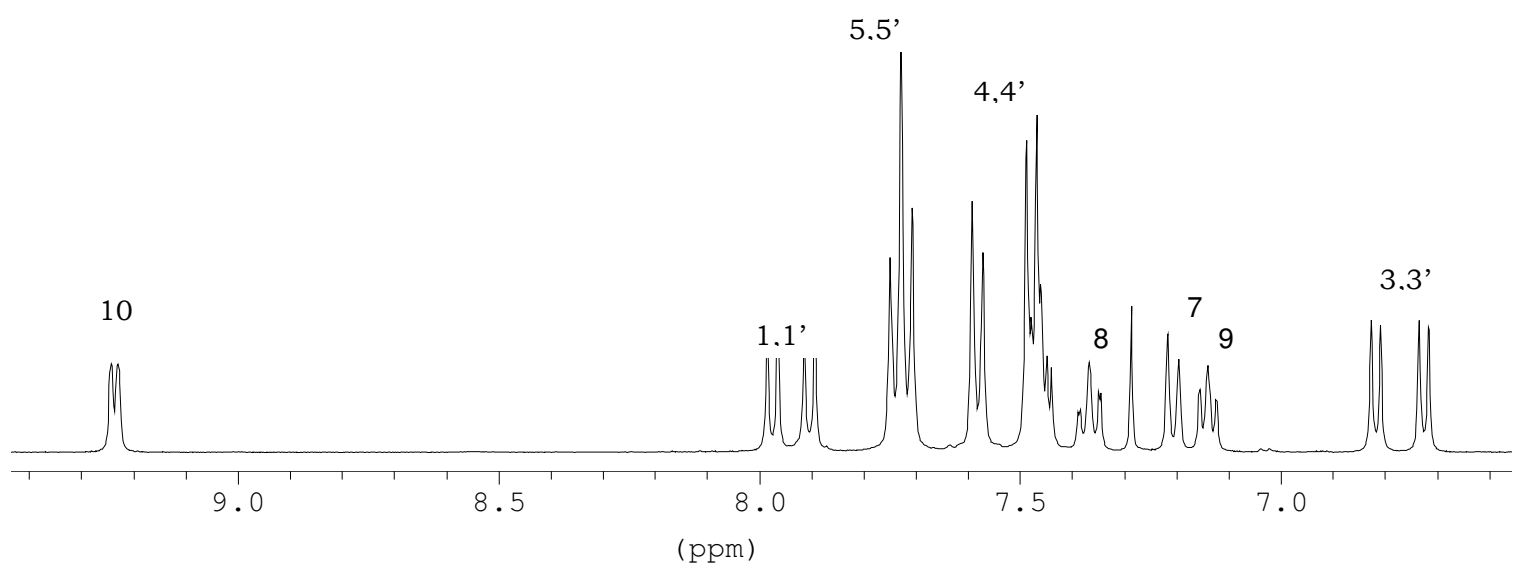

In the ${ }^{13} \mathrm{C}$ NMR spectra of $(\mathbf{3 a})$ and $(\mathbf{3 b})$, the carbon resonances for the four carbonyl ligands appeared at 225, 217 and $205 \mathrm{ppm}$ with the intensity ratio of $(1: 1: 2)$. The signal at $205 \mathrm{ppm}$ is assigned to the two axial carbonyl ligands. The proton resonance for $\mathrm{H}^{10}$ showed a downfield shift of $0.7 \mathrm{ppm}$ upon coordination to molybdenum.

The cyclometallated palladium(II) complexes (4a) - (4d) are characterised by ${ }^{1} \mathrm{H}$ and ${ }^{13} \mathrm{C}$ NMR spectroscopy and they show similar chemical shifts except for $\mathrm{H}^{10}$ and $\mathrm{H}^{11}$. (4b), (4c) and (4d) are less soluble in $\mathrm{CDCl}_{3}$ than (4a), and this limited solubility hindered performing some of the NMR experiments. The aromatic region of the ${ }^{1} \mathrm{H}$ NMR spectrum of (4a) is shown in Figure 4. In the ${ }^{1} \mathrm{H}$ NMR spectrum of (4a), $\mathrm{H}^{11}$ appeared as a doublet at $7.47 \mathrm{ppm}$ with a weak four bond coupling to $\mathrm{H}^{12},{ }^{4} \mathrm{~J}(\mathrm{HH})=1.5 \mathrm{~Hz}$. The chemical shifts of $\mathrm{H}^{10}$ and $\mathrm{H}^{11}$ showed up field shifts when the halide is changed from chloride to iodide. The peak observed at $160.4 \mathrm{ppm}$ in the ${ }^{13} \mathrm{C} \mathrm{NMR}$ spectrum of (4a) is tentatively assigned to the orthometallated carbon. In the ${ }^{1} \mathrm{H}$ NMR spectrum of (5) the allyl protons appeared as broad peaks at 3.99, 3.30, 2.45 and $1.85 \mathrm{ppm}$ and are similar to other allyl compounds reported (Ahmad et al 1996 \& Ollagnier et al 2008). 
Aromatic region of the ${ }^{1} \mathrm{H}$ NMR spectrum of (4a) with the atom labelling used for the assignment of NMR data

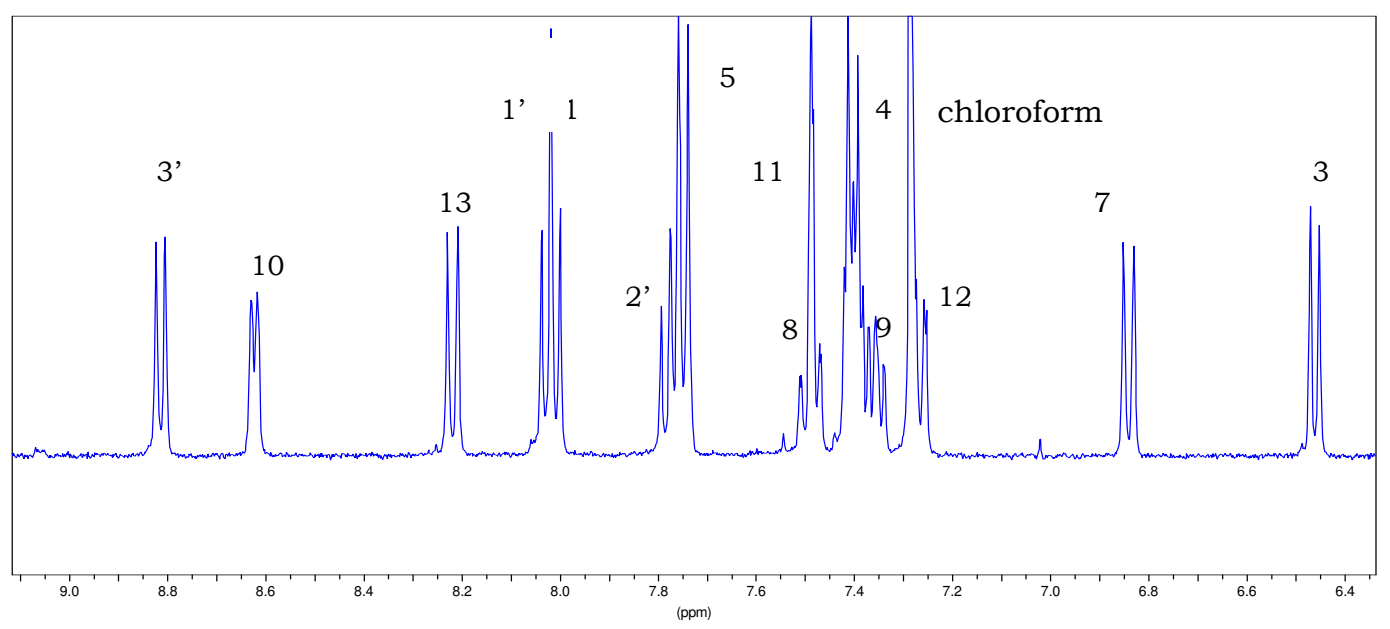

In the ${ }^{1} \mathrm{H}$ NMR spectrum of $[\mathrm{Pd}(\mathrm{DMAP})(\mathrm{L})] \mathrm{PF}_{6}(\mathbf{6 a}), \mathrm{H}^{11}$ appeared as a doublet at $6.82 \mathrm{ppm}$ with ${ }^{4} \mathrm{~J}(\mathrm{HH})=2.0 \mathrm{~Hz}$ whilst the aryl protons of DMAP gave a AB-pattern at 8.40 and $6.84 \mathrm{ppm}$ with ${ }^{3} \mathrm{~J}(\mathrm{HH})=7.0 \mathrm{~Hz}$. The phosphorus-31 resonance of $(\mathbf{6 b})$ was observed at $42.3 \mathrm{ppm}$. In the ${ }^{1} \mathrm{H}$ NMR spectrum, $\mathrm{H}^{11}$ was a doublet of doublet at $6.75 \mathrm{ppm}$ with coupling to $\mathrm{H}^{10}$ and phosphorus, ${ }^{4} \mathrm{~J}(\mathrm{PH})=6.0 \mathrm{~Hz}$.

\section{Conclusion}

We have prepared a novel ligand system based on fluoranthene which can act as a bidentate ligand through both $\mathrm{N}$-donors and an anionic terdentate NNC-ligand.

\section{Acknowledgements}

The authors are grateful to Dr. John O'Brien and M. Ruether for NMR support. S.D.P. thanks Science Foundation Ireland and CRANN for financial support. 


\section{References}

Ahmad, M., Perera, S. D., Shaw, B. L. and Thornton-Pett, M, Complexes of tert-butyl dipheylphosphinomethyl ketone $N$-phenylhydrazone, $Z$ $\mathrm{PPh}_{2} \mathrm{CH}_{2} \mathrm{C}\left(\mathrm{Bu}^{\mathrm{t}}\right)=\mathrm{NNHPh}$ with Mo, Pd or Pt. Crystal structure of cis$\left[\mathrm{PdCl}_{2}\left\{Z-\mathrm{PPh}_{2} \mathrm{CH}_{2} \mathrm{C}\left(\mathrm{Bu}^{\mathrm{t}}\right)=\mathrm{NNHPh}\right\}_{2}\right]$, Inorg. Chim. Acta, 245: 59.

Darensbourg, D. J. and Kump, R. L. (1978), A convenient synthesis of cis$\mathrm{Mo}(\mathrm{CO})_{4} \mathrm{~L}_{2}$ derivatives, Inorg. Chem., 17: 2680.

Debad, J. D., Morris, J. C., Lynch, V., Magnus, P. and Bard, A. J. (1996), Dibenzo- tetraphenylperiflanthene: Synthesis, photophysical properties, and electrogenerated chemiluminescence, J. Am. Chem. Soc., 119: 2374.

Draper, S. M., Gregg, D. J. and Madathil, R. (2002), Heterosuperbenzenes: A new family of nitrogen-functionalized, graphitic molecules, J. Am. Chem. Soc., 124: 3486-3487.

Draper, S. M., Gregg, D. J., Schofield, E. R., Browne, W. R., Duati, M., Vos, J. G. and Passaniti, P. (2004), Complexed nitrogen heterosuperbenzene: The coordinating properties of a remarkable ligand, J. Am. Chem. Soc., 126: 8694.

Hii, K. K., Perera, S. D. and Shaw, B. L. (1995), Terdentate (P-N-N) complexes of a new pyridyl azine phosphine $Z, E-\mathrm{PPh}_{2} \mathrm{CH}_{2} \mathrm{C}\left(\mathrm{Bu}^{\mathrm{t}}\right)=\mathrm{N}-\mathrm{N}=\mathrm{C}(\mathrm{Me}) \mathrm{C}_{5} \mathrm{H}_{4} \mathrm{~N}$ and its deprotonated derivative (an azo-phosphine) with transition metals, J. Chem. Soc., Dalton Trans., 624.

Ollangnier, C. M. A., Perera, S. D., Fitchett, C. M. and Draper, S. M., (2008), Rhodium and palladium complexes of a pyridyl-centred polyphenylene derivative, J. Chem. Soc. Dalton Trans., 283-290.

Sygula, A. and Rabideau, P.W. (1999), Non-Pyrolytic Syntheses of Buckybowls: Corannulene, Cyclopentacorannulene, and a Semibuckminsterfullerene, J. Am. Chem. Soc., 121: 7800.

Watson, M. D., Fechtenkotter, A., and Mullen, K. (2001), Giant polycyclic aromatic hydrocarbons, Chem. Rev., 101: 1267.

Wehmeier, M., Wagner, M. and Mullen, K. (2001), Novel Perylene chromophores obtained by a facile oxidative cyclodehydrogenation route, Chem. Eur. J., 7, 2197. 